\title{
CIRCULATORY STUDIES AT REST AND DURING EXERCISE IN COARCTATION OF THE AORTA BEFORE AND AFTER OPERATION
}

BY

\author{
S. H. TAYLOR AND K. W. DONALD* \\ From the Department of Medicine, Queen Elizabeth Hospital, University of Birmingham
}

Received April 10, 1959

In spite of the increased clinical recognition of coarctation of the aorta, there is still only a limited knowledge of the hæmodynamic disturbances in this condition. Further, although surgical correction has now become a routine and safe procedure, no comprehensive report of the circulatory adjustments after resection has yet been published.

In 1931, Blumgart et al. studied the arteriovenous blood oxygen content difference in the arms and legs of two patients with coarctation. In addition, by simultaneous brachial and femoral arterial pressure tracings they demonstrated the damping and delay of the pulse wave as it passed down the aorta. However, both patients were in heart failure and one had also pulmonary tuberculosis.

Grollman and Ferrigan (1934), Strayhorn (1936), and Lequime (1940) all found a raised or high normal resting cardiac output in single cases of coarctation of the aorta by the acetylene technique. Stewart and Bailey (1941) also studied the cardiac output of 14 patients with coarctation by the same method and reported that the resting cardiac output was normal or slightly raised. However, Grollman's patient and five of Stewart's had aortic regurgitation and two of the latter series had also clinical evidence of heart failure.

Bing et al. (1948) determined the resting cardiac output by right heart catheterization in 16 patients before and in 9 after operation. Although the mean resting cardiac output was normal both before and after operation, in three patients it was greater than $5.01 . / \mathrm{min} . / \mathrm{sq} . \mathrm{m}$. on both occasions. A significant postoperative fall of brachial arterial blood pressure without change in the cardiac output was observed in most of these patients at the time of the second studies, three weeks after operation.

Beard et al. (1951) studied 14 patients with uncomplicated coarctation by means of a dye dilution technique and intravascular pressure records. The cardiac output at rest was either normal or slightly raised in all of them before operation, but decreased in each of the six studied after operation. Many of them had brachial systolic hypertension before operation, which was reduced although still raised above normal at the time of the second studies, two weeks after aortic reconstruction. The femoral arterial mean pressures were within normal limits both before and after operation. Following resection, the delay in onset of the femoral pulse and the interval between onset and attainment of peak pressure were both decreased, supporting, the earlier observations of Brown et al. (1948) and Hallenbeck et al. (1951).

Werkö et al. (1956) have reported similar findings in seven patients with coarctation studied at rest before reconstruction of the aorta. Although the mean resting cardiac output of all seven was within normal limits, two had outputs greater than $4.81 . / \mathrm{min} . / \mathrm{sq} . \mathrm{m}$. In addition, the cardiac output and right atrial, pulmonary, and mean systemic arterial pressures were determined during exercise in two of them before and after operation. The exercising pulmonary arterial pressures were normal in both instances, but the response of the cardiac output to exercise cannot be assessed in the absence of exercising oxygen uptake cata. In both patients, moreover, the coarctation was complicated by valvular heart disease. Culbertson et al. (1957) while studying the hepatic and renal circulations in this condition, reported a high or normal resting cardiac output in eight patients and an abnormally low cardiac output in two others with left ventricular failure.

The raised blood pressure found in patients with coarctation is still unexplained. Blumgart et al. (1931) and Lewis (1933) considered it to be due to the increased total resistance of the coarctation and the collaterals. Page (1940), Steele (1941) and later Genest et al. (1948), have suggested that a renal mechanism similar to that demonstrated by Goldblatt was the probable cause of the hypertension. More recently, Harris et al. (1950) and Werkö et al. (1956) have found that the renal blood flow was normal both before and after

* Present address of bo:h authors, Department of Medicine, University of Edinburgh. 
resection of the coarctation and that such changes as were found did not correlate with the blood pressure changes. Prinzmetal and Wilson (1936) claimed to have demonstrated abnormal vasomotor behaviour in the upper limbs of patients with coarctation, but neither Pickering (1935) nor Patterson et al. (1957) have been able to confirm these observations. Pickering (1955), Graybiel et al. (1935) and Granström (1951) have all hinted at a clinical and functional dissimilarity of the hypertension in this condition and that of essential hypertension. However, apart from the absence of the development of malignant hypertension in coarctation, no other precise difference has yet been convincingly demonstrated. For this reason the findings in normal subjects (Donald et al., 1955) and patients with essential hypertension (Taylor et al., 1957), studied in this laboratory under the same conditions, are included in the results for comparison.

Crafoord and Nylin (1945) reported that the brachial arterial pressure had returned to normal levels in their first two successfully resected cases. Since that time there have been numerous reports of the return of the brachial blood pressure to "normal" after operation. Gross (1953) in fact considered that the resection was inadequate if a normal brachial arterial pressure was not achieved after operation. These early claims were not fully supported by Counihan (1956) who studied the brachial blood pressure under more controlled conditions in 20 patients after successful and adequate resection of the coarctation: he found that, although the resting blood pressure was almost invariably reduced after operation, it still remained abnormal in a large number of instances. Campbell and Baylis (1956) and Campbell (1958) reached similar conclusions.

Thus it will be seen that in previous work, only certain rather isolated aspects have been studied, almost always at rest and sometimes in patients with associated valve disease and even heart failure. This work describes the circulatory changes that occur at rest and during exercise, before and after operation, in a series of fit patients with uncomplicated coarctation of the aorta and without heart failure.

\section{METHODS}

Clinical Details. Observations were made on 20 patients with coarctation of the aorta at rest and during exercise. Clinical and laboratory details of these patients are summarized in Tables I and II respectively. In all patients the coarctation was of adult type, the constriction being at or just distal to the origin of the left subclavian artery. In 12 the diagnosis was confirmed at operation and in the remainder the diagnosis was based on the clinical and radiological findings. End-to-end anastomosis of the aorta was carried out in all except one (CN1) in whom an aortic graft was inserted. The 12 patients in whom the coarctation was resected were restudied between 6 and 18 months after operation. All patients had been in hospital, but not confined to bed, for two weeks before the study and none were receiving any medicinal therapy. The sexes are nearly equally represented $(M: F=11: 9)$ and their ages ranged from 15 to 37 years (mean 24 years). Seven of the 12 patients in whom the coarctation was resected were women. Their ages ranged from 16 to 37 years (mean 26 years) at the time of operation.

Headache was the presenting symptom in 8 of the 20 patients. Four complained of leg cramps on exercise, and in one of them it was the presenting symptom. The remaining 11 were symptom-free and had been referred when abnormal physical signs had been discovered during routine antenatal, insurance, factory, or mass-radiograph examinations. No patient complained directly of breathlessness on exertion. The resting brachial arterial pressure was recorded by mercury sphygmomanometry, usually sitting but occasionally reclining, in each patient every two hours for 72 hours immediately before the study and the range of readings is shown in Table I. No patient had any clinical evidence of valvular or other heart disease and all were in sinus rhythm. The femoral pulses were absent or barely palpable before operation in all. At the time of the post-operative second study the femoral and more distal pulses were strongly palpable in all patients. Synchronous brachial and femoral arterial tracings were performed on five (CN1, CN3, CN7, CN10, and CN17) before and after operation. All demonstrated a reduction in delay of onset of the femoral pulse and a greatly increased femoral pulse pressure after operation. Before operation only one (CN3) had certain but moderate left ventricular enlargement, as judged clinically and radiologically: this had decreased considerably 18 months after operation at the time of the second study. Three others (CN1, CN7, and CN14) had a radiological cardiac configuration suggesting left ventricular hypertrophy without dilatation: this had disappeared 18 months after operation in one (CN14), and was unchanged in the other two. Eight (CN1, CN2, CN3, CN7, CN10, CN14, CN17, and CN19) showed electrocardiographic evidence of left ventricular hypertrophy. In seven of these the coarctation was resected and after operation four (CN1, CN7, CN10, and CN14) had normal records and in three the pattern was unchanged; one 
TABLE I

Clinical Details of Patients Studied

\begin{tabular}{|c|c|c|c|c|c|c|c|}
\hline \multirow[t]{2}{*}{ Patient } & \multirow[t]{2}{*}{ Sex } & \multirow[t]{2}{*}{$\begin{array}{l}\text { Before } \\
\text { or after } \\
\text { operation }\end{array}$} & \multirow[t]{2}{*}{$\begin{array}{l}\text { Age } \\
\text { yr. }\end{array}$} & \multirow[t]{2}{*}{$\begin{array}{l}\text { Surface } \\
\text { area } \\
\text { sq. } \mathrm{m} .\end{array}$} & \multirow[t]{2}{*}{ Symptoms and duration } & \multicolumn{2}{|c|}{$\begin{array}{l}\text { Range of resting } \\
\text { brachial arterial } \\
\text { pressure } \mathrm{mm} . \mathrm{Hg}\end{array}$} \\
\hline & & & & & & Systolic & Diastolic \\
\hline CN1 & $\mathbf{F}$ & Before & 26 & $1 \cdot 23$ & Headaches -7 yr. Leg cramps -5 yr. & $220-250$ & $110-120$ \\
\hline $\mathrm{CN} 2$ & $\mathbf{F}$ & $\begin{array}{l}\text { After } \\
\text { Before }\end{array}$ & $\begin{array}{l}28 \\
34\end{array}$ & $\begin{array}{l}1 \cdot 31 \\
1.67\end{array}$ & $\begin{array}{l}\text { None } \\
\text { Headaches }-20 \mathrm{yr} \text {. }\end{array}$ & $\begin{array}{l}140-160 \\
160-180\end{array}$ & $\begin{array}{r}80-100 \\
100-110\end{array}$ \\
\hline & & & 36 & 1.67 & Unchanged & $160-170$ & 100-110 \\
\hline $\mathrm{CN} 3$ & $\mathbf{F}$ & Before & 32 & $1 \cdot 36$ & Headaches -5 yr. & $210-300$ & $110-140$ \\
\hline & & After & 34 & $1 \cdot 35$ & None & $160-210$ & $110-120$ \\
\hline CN4 & $\mathbf{F}$ & Before & 16 & $1 \cdot 61$ & Headaches-1 yr. & $180-190$ & $80-100$ \\
\hline & & After & 17 & 1.68 & None & $140-160$ & $90-100$ \\
\hline CN5 & $\mathbf{F}$ & *Before & 25 & $1 \cdot 71$ & None & $150-160$ & $100-110$ \\
\hline CN6 & $\mathbf{F}$ & $\begin{array}{l}\text { Before } \\
\text { After }\end{array}$ & $\begin{array}{l}22 \\
22\end{array}$ & $\begin{array}{l}1.52 \\
1.51\end{array}$ & $\begin{array}{l}\text { Headaches }-5 \text { yr. Leg cramps }-3 \text { yr. } \\
\text { Leg cramps unchanged }\end{array}$ & $\begin{array}{l}180-230 \\
130-150\end{array}$ & $\begin{array}{c}100-110 \\
80-90\end{array}$ \\
\hline CN7 & $\mathbf{F}$ & Before & 24 & $1 \cdot 52$ & None & $230-200$ & $120-130$ \\
\hline $\mathrm{CN8}$ & F & $\begin{array}{l}\text { After } \\
\text { Before }\end{array}$ & $\begin{array}{l}24 \\
29\end{array}$ & $\begin{array}{l}1.53 \\
1.69\end{array}$ & $\begin{array}{l}\text { None } \\
\text { Headaches- }\end{array}$ & $150-160$ & $90-100$ \\
\hline CNO & $F$ & $\begin{array}{l}\text { Berore } \\
\text { After }\end{array}$ & $\begin{array}{l}29 \\
31\end{array}$ & $\begin{array}{l}1.69 \\
1.66\end{array}$ & $\begin{array}{l}\text { Headaches }-3 \mathrm{yr} \text {. Leg } \\
\text { Leg cramps unchanged }\end{array}$ & $\begin{array}{l}180-200 \\
140-150\end{array}$ & $\begin{array}{l}100-110 \\
80-90\end{array}$ \\
\hline CN9 & $\mathbf{M}$ & Before & 18 & $1 \cdot 52$ & None & $160-200$ & $100-110$ \\
\hline CN10 & $\mathbf{M}$ & $\begin{array}{l}\text { After } \\
\text { Before }\end{array}$ & $\begin{array}{l}20 \\
37\end{array}$ & $\begin{array}{l}1 \cdot 84 \\
1.89\end{array}$ & $\begin{array}{l}\text { None } \\
\text { Headaches-1 yr. Palpitation-1 yr. }\end{array}$ & $\begin{array}{l}140-160 \\
180-200\end{array}$ & $\begin{array}{c}80-90 \\
100-110\end{array}$ \\
\hline & & After & 37 & 1.90 & None & $150-170$ & $90-100$ \\
\hline CN11 & $\mathbf{M}$ & *Before & 15 & $1 \cdot 65$ & None & $160-180$ & $80-110$ \\
\hline CN12 & $\mathbf{M}$ & Before & 17 & 1.78 & Leg cramps- -3 yr. & $180-200$ & $110-120$ \\
\hline & & & 18 & 1.80 & Leg cramps & 160-170 & $90-100$ \\
\hline CN13 & M & *Before & 17 & $1 \cdot 72$ & None & $160-180$ & $80-100$ \\
\hline $\mathrm{CN} 14$ & $\mathbf{M}$ & $\begin{array}{l}\text { Before } \\
\text { After }\end{array}$ & $\begin{array}{l}35 \\
35\end{array}$ & $\begin{array}{l}1.62 \\
1.62\end{array}$ & $\begin{array}{l}\text { Headaches-1 yr. } \\
\text { None }\end{array}$ & $\begin{array}{l}200-220 \\
130-150\end{array}$ & $\begin{array}{r}115-130 \\
80-100\end{array}$ \\
\hline CN15 & $\mathbf{F}$ & *Before & 20 & 1.80 & None & $140-160$ & $70-90$ \\
\hline & $\mathbf{M}$ & *Before & 18 & 1.87 & None & $180-190$ & $70-80$ \\
\hline CN17 & $\mathbf{M}$ & Before & 16 & 1.83 & None & $210-260$ & $110-135$ \\
\hline & & & 16 & 2.06 & None & $170-190$ & $100-110$ \\
\hline CN18 & $\mathbf{M}$ & *Before & 30 & 1.65 & None & -230 & $110-130$ \\
\hline CN19 & $\mathbf{M}$ & * Befo & 18 & 1.95 & None & & $110-130$ \\
\hline CN20 & $\mathbf{M}$ & ${ }^{*}$ Before & 28 & 1.79 & None & $170-200$ & $110-120$ \\
\hline
\end{tabular}

* These 8 patients have not had an operation.

(CN19) was not restudied. Radiological rib notching was present in all patients and remained unchanged during the 18 months following operation. The optic fundi were normal in all and in none was there any proteinuria or other abnormal urinary constituents. All were able to concentrate urine to a specific gravity greater than 1022 after nine hours water deprivation and their creatinine clearances were also all within normal limits both before and after operation. Intravenous excretory pyelograms were normal in all. The blood hæmoglobin concentration was within normal limits in all patients at the time of both studies.

In view of the current controversy concerning the risks of pregnancy in coarctation of the aorta (Goodwin, 1958), it is of interest that four of the six married women had each had two normal pregnancies without difficulty before operation. The remaining two women had normal pregnancies subsequent to operation.

Laboratory Techniques. All patients were investigated in the fasting condition and each performed leg exercise in the supine position on a constant-speed variable-load bicycle type of ergometer. The patient's ventilation, oxygen uptake, and arterial and mixed venous blood oxygen saturations were measured at rest and during the fifth minute of leg exercise carried out at a constant speed and set load. In nine patients the study was repeated at one or two further levels of exercise, after a rest period of 20 minutes between each study. Arterial samples were withdrawn and pressures recorded from an indwelling needle in the brachial artery. The expired air was collected in a Tissot spirometer and intravascular pressures were measured with capacitance manometers and recorded on a Sanborn Poly Viso. The zero reference level was fixed at $10 \mathrm{~cm}$. above the plane of the X-ray table and in each subject the height of the sternal angle above the table was also recorded. Pulsatile pressures were measured at the end of expiration and mean pressures throughout the respiratory cycle. Pulmonary wedge (pulmonary "capillary") pressures were obtained by impacting 
TABLE II

Observations at Rest aNd DURING Exercise

Before and After Operation in Patients with Coarctation of the Aorta

\begin{tabular}{|c|c|c|c|c|c|c|c|c|c|c|c|c|c|c|c|c|c|c|c|c|c|c|c|c|c|}
\hline \multirow{4}{*}{ Patient } & \multirow{4}{*}{ State } & \multirow{3}{*}{\multicolumn{2}{|c|}{$\begin{array}{c}\text { Oxygen } \\
\text { uptake } \\
\text { ml./min./ } \\
\text { sq.m. } \\
\text { NTP }\end{array}$}} & \multirow{3}{*}{\multicolumn{2}{|c|}{$\begin{array}{c}\text { Cardiac } \\
\text { output } \\
\text { l./min./ } \\
\text { sq.m. }\end{array}$}} & \multirow{3}{*}{\multicolumn{2}{|c|}{$\begin{array}{c}\text { Heart } \\
\text { rate } \\
\text { per min. }\end{array}$}} & \multirow{3}{*}{\multicolumn{2}{|c|}{$\begin{array}{c}\text { Ventilation } \\
\text { 1./min./ } \\
\text { sq.m. } \\
\text { NTP }\end{array}$}} & \multicolumn{16}{|c|}{ Pressures mm. $\mathbf{H g}$} \\
\hline & & & & & & & & & & \multirow{2}{*}{\multicolumn{2}{|c|}{$\begin{array}{c}\text { Right } \\
\text { atrial }\end{array}$}} & \multicolumn{6}{|c|}{ Pulmonary arterial } & \multirow{2}{*}{\multicolumn{2}{|c|}{$\begin{array}{c}\text { Pul- } \\
\text { mon- } \\
\text { ary } \\
\text { wedge }\end{array}$}} & \multicolumn{6}{|c|}{ Brachial arterial } \\
\hline & & & & & & & & & & & & \multicolumn{2}{|c|}{ Sys. } & \multicolumn{2}{|c|}{ Dias. } & \multicolumn{2}{|c|}{ Mean } & & & \multicolumn{2}{|c|}{ Sys. } & \multicolumn{2}{|c|}{ Dias. } & \multicolumn{2}{|c|}{ Mean } \\
\hline & & B & $\mathbf{A}$ & & $\mathbf{A}$ & $\mathbf{B}$ & $\mathbf{A}$ & B & $\mathbf{A}$ & B & $\mathbf{A}$ & B & $\mathbf{A}$ & B & $\mathbf{A}$ & B & $\mathbf{A}$ & $\mathbf{B}$ & $\mathbf{A}$ & B & $\mathbf{A}$ & B & $\mathbf{A}$ & $\mathbf{B}$ & $\mathbf{A}$ \\
\hline CN1 & & & & & & $\begin{array}{l}120 \\
134\end{array}$ & $\begin{array}{l}120 \\
150\end{array}$ & & & -4 & 4 & $\begin{array}{l}30 \\
32\end{array}$ & $\begin{array}{l}30 \\
38\end{array}$ & $\begin{array}{ll}26 & 1 \\
22 & 3\end{array}$ & $\begin{array}{l}15 \\
30\end{array}$ & $\begin{array}{l}27 \\
25\end{array}$ & $\begin{array}{l}17 \\
27\end{array}$ & $\begin{array}{l}12 \\
20\end{array}$ & $\begin{array}{r}7 \\
14\end{array}$ & & & & $\begin{array}{l}90 \\
90\end{array}$ & $\begin{array}{l}157 \\
161\end{array}$ & $\begin{array}{l}110 \\
115\end{array}$ \\
\hline $\mathrm{CN} 2$ & Rest & $\begin{array}{l}115 \\
327\end{array}$ & $\begin{array}{l}122 \\
321\end{array}$ & & & $\begin{array}{r}94 \\
142\end{array}$ & $\begin{array}{r}90 \\
140 \\
\end{array}$ & & & 5 & $\begin{array}{l}6 \\
4\end{array}$ & $\begin{array}{l}28 \\
50\end{array}$ & $\begin{array}{l}20 \\
38\end{array}$ & \begin{tabular}{l|l}
13 & 1 \\
27 & 2
\end{tabular} & $\begin{array}{l}14 \\
27\end{array}$ & $\begin{array}{l}16 \\
31\end{array}$ & $\begin{array}{l}15 \\
28\end{array}$ & $\begin{array}{l}12 \\
18\end{array}$ & $\begin{array}{l}10 \\
15\end{array}$ & & & & & & \\
\hline $\mathrm{CN} 3$ & $\begin{array}{l}\text { Rest } \\
\text { Ex. 1* } \\
\text { Ex. } 2\end{array}$ & $\begin{array}{l}150 \\
541 \\
734\end{array}$ & $\begin{array}{l}153 \\
603 \\
706\end{array}$ & & $\begin{array}{l}4 \cdot 23 \\
6 \cdot 72 \\
7 \cdot 32\end{array}$ & $\begin{array}{r}90 \\
168 \\
184\end{array}$ & $\begin{array}{r}90 \\
148 \\
162\end{array}$ & $\begin{array}{r}3 \cdot 73 \\
13 \cdot 14 \\
15 \cdot 10\end{array}$ & & $\begin{array}{r}4 \\
4 \\
-\end{array}$ & $\begin{array}{r}4 \\
4 \\
-\end{array}$ & $\begin{array}{l}25 \\
40 \\
50\end{array}$ & $\begin{array}{l}23 \\
47 \\
51\end{array}$ & \begin{tabular}{l|l}
15 & 1 \\
33 & 3 \\
27 & 3
\end{tabular} & $\begin{array}{l}16 \\
32 \\
34\end{array}$ & $\begin{array}{l}17 \\
31 \\
33\end{array}$ & $\begin{array}{l}16 \\
34 \\
38\end{array}$ & $\begin{array}{r}9 \\
22 \\
24\end{array}$ & $\begin{array}{l}10 \\
23 \\
26\end{array}$ & & & $\begin{array}{l}144 \\
180 \\
180\end{array}$ & & & $\begin{array}{l}123 \\
153 \\
161\end{array}$ \\
\hline CN4 & & & & & $\begin{array}{l}05 \\
24\end{array}$ & $\begin{array}{r}96 \\
144\end{array}$ & $\begin{array}{r}84 \\
160 \\
\end{array}$ & $\begin{array}{r}2 \cdot 59 \\
14 \cdot 28\end{array}$ & 0 & 2 & $\begin{array}{l}4 \\
5\end{array}$ & $\begin{array}{l}30 \\
27\end{array}$ & $\begin{array}{l}23 \\
32\end{array}$ & \begin{tabular}{l|l}
22 & 1 \\
21 & 2
\end{tabular} & $\begin{array}{l}16 \\
24\end{array}$ & $\begin{array}{l}20 \\
21\end{array}$ & $\begin{array}{l}16 \\
22\end{array}$ & $\begin{array}{l}7 \\
8\end{array}$ & $\begin{array}{r}7 \\
10\end{array}$ & & & $\begin{array}{l}100 \\
118\end{array}$ & & $\begin{array}{l}27 \\
47\end{array}$ & $\begin{array}{l}123 \\
141\end{array}$ \\
\hline & & & - & & - & $\begin{array}{r}84 \\
160\end{array}$ & - & $\begin{array}{r}6 \cdot 60 \\
10 \cdot 55\end{array}$ & - & $\begin{array}{l}4 \\
4\end{array}$ & - & $\begin{array}{l}24 \\
42\end{array}$ & - & $\begin{array}{l}16 \\
31\end{array}$ & - & $\begin{array}{l}18 \\
30\end{array}$ & - & $\begin{array}{l}10 \\
14\end{array}$ & 二 & & 二 & $\begin{array}{l}108 \\
126\end{array}$ & - & $\begin{array}{l}124 \\
154\end{array}$ & - \\
\hline CN6 & & & $\begin{array}{l}134 \\
599\end{array}$ & & $\begin{array}{l}3 \cdot 34 \\
6 \cdot 14\end{array}$ & $\begin{array}{r}82 \\
136\end{array}$ & $\begin{array}{r}84 \\
116 \\
\end{array}$ & & $\begin{array}{r}3.05 \\
14.96\end{array}$ & $\begin{array}{l}3 \\
7\end{array}$ & $\begin{array}{l}4 \\
6\end{array}$ & $\begin{array}{l}20 \\
40\end{array}$ & $\begin{array}{l}20 \\
38\end{array}$ & $\begin{array}{l}15 \\
34\end{array}$ & $\begin{array}{l}13 \\
23\end{array}$ & $\begin{array}{l}16 \\
32\end{array}$ & $\begin{array}{l}14 \\
24\end{array}$ & $\begin{array}{l}10 \\
13\end{array}$ & $\begin{array}{r}9 \\
14\end{array}$ & & $\begin{array}{l}150 \\
190\end{array}$ & $\begin{array}{l}106 \\
125\end{array}$ & $\begin{array}{r}90 \\
104\end{array}$ & & \\
\hline CN7 & & & $\begin{array}{l}118 \\
665\end{array}$ & & & $\begin{array}{r}96 \\
176\end{array}$ & \begin{tabular}{|l|}
116 \\
182 \\
\end{tabular} & & $\begin{array}{r}2 \cdot 69 \\
15 \cdot 16\end{array}$ & $\frac{4}{-}$ & $\begin{array}{l}3 \\
5\end{array}$ & $\begin{array}{l}40 \\
76\end{array}$ & $\begin{array}{l}30 \\
70\end{array}$ & $\begin{array}{l}20 \\
54\end{array}$ & $\begin{array}{l}20 \\
50\end{array}$ & $\begin{array}{l}23 \\
56\end{array}$ & $\begin{array}{l}22 \\
45\end{array}$ & $\begin{array}{l}12 \\
51\end{array}$ & $\begin{array}{r}9 \\
14\end{array}$ & & $\begin{array}{l}174 \\
200\end{array}$ & $\begin{array}{l}130 \\
156\end{array}$ & & & \\
\hline CN8 & & & $\begin{array}{l}133 \\
507\end{array}$ & & $\begin{array}{l}2.79 \\
5 \cdot 11\end{array}$ & $\begin{array}{r}80 \\
128\end{array}$ & \begin{tabular}{|r|}
84 \\
114 \\
\end{tabular} & $\begin{array}{r}3.90 \\
13.47\end{array}$ & $\begin{array}{r}3 \cdot 73 \\
11 \cdot 34\end{array}$ & $\frac{5}{-}$ & 3 & $\begin{array}{l}25 \\
28\end{array}$ & $\begin{array}{l}30 \\
46\end{array}$ & $\begin{array}{l}14 \\
20\end{array}$ & $\begin{array}{l}13 \\
27\end{array}$ & $\begin{array}{l}16 \\
18\end{array}$ & $\begin{array}{l}14 \\
22\end{array}$ & $\begin{array}{l}11 \\
15\end{array}$ & $\begin{array}{r}6 \\
12\end{array}$ & & $\begin{array}{l}140 \\
170\end{array}$ & $\begin{array}{l}110 \\
135\end{array}$ & $\begin{array}{r}90 \\
100\end{array}$ & & \\
\hline CN9 & & & $\begin{array}{l}159 \\
584\end{array}$ & & & $\begin{array}{r}84 \\
126\end{array}$ & $\begin{array}{r}64 \\
100 \\
\end{array}$ & & $\begin{array}{l}3 \cdot 16 \\
8 \cdot 56\end{array}$ & $\begin{array}{l}3 \\
4\end{array}$ & $\begin{array}{l}2 \\
4\end{array}$ & $\begin{array}{l}16 \\
23\end{array}$ & $\begin{array}{l}25 \\
27\end{array}$ & \begin{tabular}{l|}
11 \\
17
\end{tabular} & $\begin{array}{l}18 \\
19\end{array}$ & $\begin{array}{l}13 \\
16\end{array}$ & $\begin{array}{l}15 \\
16\end{array}$ & $\begin{array}{l}8 \\
7\end{array}$ & $\begin{array}{r}8 \\
10\end{array}$ & & & $\begin{array}{l}110 \\
120\end{array}$ & $\begin{array}{l}80 \\
74\end{array}$ & $\begin{array}{l}137 \\
157\end{array}$ & $\begin{array}{l}100 \\
104\end{array}$ \\
\hline CN10 & & & $\begin{array}{l}130 \\
685\end{array}$ & & & $\begin{array}{r}80 \\
126\end{array}$ & $\begin{array}{r}84 \\
130 \\
\end{array}$ & & \begin{tabular}{r|}
$4 \cdot 75$ \\
$10 \cdot 20$
\end{tabular} & $\begin{array}{l}5 \\
8\end{array}$ & $\begin{array}{l}4 \\
7\end{array}$ & $\begin{array}{l}21 \\
47\end{array}$ & $\begin{array}{l}24 \\
46\end{array}$ & $\begin{array}{l}16 \\
35\end{array}$ & $\begin{array}{l}14 \\
32\end{array}$ & $\begin{array}{l}16 \\
31\end{array}$ & $\begin{array}{l}16 \\
29\end{array}$ & $\begin{array}{l}14 \\
26\end{array}$ & $\begin{array}{l}11 \\
14\end{array}$ & & & $\begin{array}{l}100 \\
124\end{array}$ & $\begin{array}{r}90 \\
110\end{array}$ & $\begin{array}{l}127 \\
193\end{array}$ & $\begin{array}{l}111 \\
147\end{array}$ \\
\hline CN11 & $\begin{array}{l}\text { Rest } \\
\text { Ex. }\end{array}$ & $\begin{array}{l}178 \\
956\end{array}$ & 二 & & - & $\begin{array}{r}78 \\
126\end{array}$ & 二 & $\begin{array}{r}3.92 \\
20.67\end{array}$ & 二 & 3 & - & $\begin{array}{r}12 \\
7\end{array}$ & 二 & \begin{tabular}{l|l}
7 & - \\
2 & -
\end{tabular} & - & $\begin{array}{l}9 \\
5\end{array}$ & 二 & $\begin{array}{l}5 \\
4\end{array}$ & - & & - & $\begin{array}{r}84 \\
104\end{array}$ & - & $\begin{array}{l}109 \\
143\end{array}$ & - \\
\hline CN12 & $\begin{array}{l}\text { Rest } \\
\text { Ex. }\end{array}$ & $\begin{array}{r}185 \\
1,106\end{array}$ & $\begin{array}{r}167 \\
1,070\end{array}$ & & $\begin{array}{l}4 \cdot 60 \\
9 \cdot 01\end{array}$ & $\begin{array}{r}88 \\
174\end{array}$ & \begin{tabular}{|r|}
79 \\
162 \\
\end{tabular} & $\begin{array}{r}3.17 \\
27.03\end{array}$ & $\begin{array}{r}4 \cdot 21 \\
24 \cdot 20\end{array}$ & 8 & 4 & $\begin{array}{ll}27 \\
30 & 3\end{array}$ & $\begin{array}{l}26 \\
32\end{array}$ & $\begin{array}{l}20 \\
20\end{array}$ & 18 & $\begin{array}{l}21 \\
23\end{array}$ & $\begin{array}{l}18 \\
24\end{array}$ & $\begin{array}{l}12 \\
17\end{array}$ & $\begin{array}{r}9 \\
14\end{array}$ & & $\begin{array}{l}160 \\
235\end{array}$ & $\begin{array}{l}120 \\
132\end{array}$ & & $\begin{array}{l}147 \\
172\end{array}$ & $\begin{array}{l}120 \\
151\end{array}$ \\
\hline & $\begin{array}{l}\text { Rest } \\
\text { Ex. 1* } \\
\text { Ex. } 2\end{array}$ & $\begin{array}{l}117 \\
367 \\
772\end{array}$ & E & & - & $\begin{array}{r}76 \\
96 \\
152\end{array}$ & E & $\begin{array}{r}3 \cdot 29 \\
8 \cdot 03 \\
15 \cdot 57\end{array}$ & E & $\begin{array}{l}4 \\
6 \\
8\end{array}$ & $\overline{-}$ & $\begin{array}{l}28 \\
32 \\
42\end{array} \mid$ & - & $\begin{array}{l}18 \\
22 \\
28\end{array}$ & - & $\begin{array}{l}17 \\
20 \\
30\end{array}$ & - & $\begin{array}{l}12 \\
13 \\
18\end{array}$ & - & $\begin{array}{l}100 \\
184 \\
248\end{array}$ & E & $\begin{array}{r}80 \\
90 \\
120\end{array}$ & E & & E \\
\hline CN14 & $\begin{array}{l}\text { Rest } \\
\text { Ex. } 1 * \\
\text { Ex. } 2\end{array}$ & $\begin{array}{l}156 \\
440 \\
772\end{array}$ & $\begin{array}{l}147 \\
410 \\
668\end{array}$ & & $\begin{array}{l}2 \cdot 88 \\
5 \cdot 13 \\
7 \cdot 26\end{array}$ & $\begin{array}{l}100 \\
118 \\
146\end{array}$ & $\begin{array}{l}100 \\
126 \\
160\end{array}$ & $\begin{array}{r}3.78 \\
8.99 \\
19.86\end{array}$ & $\begin{array}{r}4 \cdot 1 \\
7 \cdot 0 \\
17 \cdot 0\end{array}$ & $\begin{array}{l}3 \\
5 \\
5\end{array}$ & $\begin{array}{l}3 \\
3 \\
4\end{array}$ & $\begin{array}{l}22 \\
40 \\
45\end{array}$ & $\begin{array}{l}16 \\
22 \\
30\end{array}$ & $\begin{array}{l}15 \\
26 \\
35\end{array}$ & $\begin{array}{l}10 \\
16 \\
23\end{array}$ & $\begin{array}{l}16 \\
26 \\
30\end{array}$ & $\begin{array}{l}10 \\
16 \\
20\end{array}$ & \begin{tabular}{l|}
10 \\
18 \\
21
\end{tabular} & $\begin{array}{r}6 \\
8 \\
10\end{array}$ & & $\begin{array}{l}130 \\
180 \\
220\end{array}$ & $\begin{array}{l}124 \\
130 \\
152\end{array}$ & $\begin{array}{r}80 \\
100 \\
138\end{array}$ & $\begin{array}{l}153 \\
173 \\
205\end{array}$ & $\begin{array}{r}97 \\
127 \\
165\end{array}$ \\
\hline & $\begin{array}{l}\text { Rest } \\
\text { Ex. 1* } \\
\text { Ex. 2* } \\
\text { Ex. } 3\end{array}$ & $\begin{array}{l}162 \\
454 \\
614 \\
694\end{array}$ & E & $\begin{array}{l}7 \cdot 00 \\
7 \cdot 67 \\
7.94\end{array}$ & E & $\begin{array}{l}100 \\
138 \\
156 \\
174\end{array}$ & E & $\begin{array}{r}3 \cdot 18 \\
8 \cdot 90 \\
12 \cdot 20 \\
14 \cdot 66\end{array}$ & E & $\begin{array}{l}2 \\
2 \\
4 \\
5\end{array}$ & $\bar{z}$ & $\left|\begin{array}{l}23 \\
22 \\
27 \\
35\end{array}\right|$ & $\bar{z}$ & $\begin{array}{l}10 \\
11 \\
20 \\
25\end{array}$ & $\bar{z}$ & $\begin{array}{l}12 \\
15 \\
19 \\
23\end{array}$ & $\bar{z}$ & $\begin{array}{r}4 \\
7 \\
11 \\
14\end{array}$ & E & & E & $\begin{array}{l}85 \\
90 \\
90 \\
90\end{array}$ & E & & E \\
\hline CN16 & $\begin{array}{l}\text { Rest } \\
\text { Ex. 1* } \\
\text { Ex. 2* } \\
\text { Ex. } 3\end{array}$ & $\begin{array}{l}67 \\
48 \\
33 \\
67\end{array}$ & z & & E & $\begin{array}{r}84 \\
108 \\
130 \\
168\end{array}$ & E & $\begin{array}{r}3 \cdot 14 \\
5 \cdot 77 \\
10 \cdot 58 \\
19 \cdot 61\end{array}$ & E & $\begin{array}{l}2 \\
3 \\
4 \\
4\end{array}$ & E & $\begin{array}{l}17 \\
22 \\
28 \\
38\end{array}$ & E & $\begin{array}{r}9 \\
15 \\
21 \\
28\end{array}$ & $\begin{array}{l}- \\
- \\
-\end{array}$ & $\begin{array}{l}10 \\
15 \\
19 \\
24\end{array}$ & - & $\begin{array}{r}4 \\
7 \\
13 \\
16\end{array}$ & - & $\begin{array}{l}180 \\
220 \\
256 \\
315\end{array}$ & E & $\begin{array}{r}70 \\
70 \\
90 \\
100\end{array}$ & E & $\begin{array}{l}107 \\
120 \\
145 \\
172\end{array}$ & E \\
\hline CN17 & $\begin{array}{l}\text { Rest } \\
\text { Ex. 1* } \\
\text { Ex. 2* } \\
\text { Ex. } 3\end{array}$ & & $\begin{array}{l}176 \\
386 \\
571 \\
778\end{array}$ & & $\begin{array}{l}5 \cdot 28 \\
6 \cdot 16 \\
7 \cdot 83 \\
8 \cdot 17\end{array}$ & $\begin{array}{r}70 \\
100 \\
126 \\
150\end{array}$ & $\begin{array}{r}80 \\
96 \\
122 \\
150\end{array}$ & $\begin{array}{r}3 \cdot 75 \\
6.96 \\
10 \cdot 08 \\
15 \cdot 16\end{array}$ & $\begin{array}{r}3 \cdot 62 \\
7 \cdot 54 \\
10 \cdot 31 \\
14 \cdot 54\end{array}$ & $\begin{array}{l}3 \\
4 \\
6\end{array}$ & $\begin{array}{l}2 \\
3 \\
3 \\
5\end{array}$ & $\begin{array}{l}32 \\
32 \\
32 \\
35\end{array}$ & $\begin{array}{l}30 \\
40 \\
45 \\
48\end{array}$ & $\begin{array}{l}23 \\
23 \\
24 \\
28\end{array}$ & $\begin{array}{l}20 \\
25 \\
28 \\
32\end{array}$ & $\begin{array}{l}25 \\
26 \\
27 \\
30\end{array}$ & $\begin{array}{l}21 \\
27 \\
30 \\
33\end{array}$ & $\begin{array}{l}10 \\
11 \\
13 \\
16\end{array}$ & $\begin{array}{l}10 \\
11 \\
12 \\
14\end{array}$ & & $\begin{array}{l}170 \\
190 \\
210 \\
270\end{array}$ & $\begin{array}{l}108 \\
110 \\
120 \\
130\end{array}$ & $\begin{array}{r}96 \\
100 \\
108 \\
112\end{array}$ & & $\begin{array}{l}121 \\
130 \\
142 \\
165\end{array}$ \\
\hline CN18 & $\begin{array}{l}\text { Rest } \\
\text { Ex. 1* } \\
\text { Ex. 2* } \\
\text { Ex. } 3\end{array}$ & $\begin{array}{l}174 \\
427 \\
657 \\
895\end{array}$ & E & $\begin{array}{l}4 \\
5 \\
7 \\
8\end{array}$ & E & $\begin{array}{r}96 \\
108 \\
134 \\
160\end{array}$ & E & $\begin{array}{r}3 \cdot 37 \\
7 \cdot 25 \\
11 \cdot 27 \\
14 \cdot 55\end{array}$ & E & - & 二 & $\begin{array}{l}30 \\
46 \\
58 \\
70\end{array}$ & - & $\begin{array}{l}15 \\
22 \\
32 \\
50\end{array}$ & $\begin{array}{l}- \\
-\end{array}$ & $\begin{array}{l}17 \\
28 \\
36 \\
40\end{array}$ & E & $\begin{array}{r}8 \\
15 \\
22 \\
28\end{array}$ & - & $\begin{array}{l}210 \\
240 \\
256 \\
286\end{array}$ & E & $\begin{array}{l}120 \\
126 \\
146 \\
156\end{array}$ & E & $\begin{array}{l}150 \\
164 \\
183 \\
199\end{array}$ & E \\
\hline & $\begin{array}{l}\text { Rest } \\
\text { Ex. 1* } \\
\text { Ex. 2* } \\
\text { Ex. 3 }\end{array}$ & $\begin{array}{l}170 \\
359 \\
553 \\
856\end{array}$ & 二 & $\begin{array}{l}4 \cdot 54 \\
5 \cdot 45 \\
6 \cdot 81 \\
8 \cdot 32\end{array}$ & E & $\begin{array}{r}86 \\
108 \\
130 \\
155\end{array}$ & E & $\begin{array}{r}3.41 \\
6.55 \\
11.06 \\
17.61\end{array}$ & E & $\frac{5}{-}$ & 二 & $\begin{array}{l}42 \\
55 \\
60 \\
64\end{array}$ & - & $\begin{array}{l}25 \\
30 \\
34 \\
40\end{array}$ & - & $\begin{array}{l}27 \\
38 \\
40 \\
42\end{array}$ & E & $\begin{array}{l}14 \\
25 \\
30 \\
36\end{array}$ & E & $\begin{array}{l}220 \\
250 \\
276 \\
320\end{array}$ & E & $\begin{array}{l}120 \\
130 \\
134 \\
140\end{array}$ & E & $\begin{array}{l}153 \\
170 \\
181 \\
200\end{array}$ & E \\
\hline CN20 & $\begin{array}{l}\text { Rest } \\
\text { Ex. 1* } \\
\text { Ex. 2* } \\
\text { Ex. } 3\end{array}$ & $\begin{array}{l}173 \\
402 \\
608 \\
896\end{array}$ & I & $\begin{array}{l}4 \cdot 24 \\
5 \cdot 16 \\
6 \cdot 27 \\
7 \cdot 75\end{array}$ & E & $\begin{array}{r}94 \\
116 \\
135 \\
168\end{array}$ & $=$ & $\begin{array}{r}5 \cdot 22 \\
7.18 \\
11.07 \\
18.70\end{array}$ & E & $\frac{5}{-}$ & E & $\begin{array}{l}25 \\
34 \\
37 \\
50\end{array}$ & - & $\begin{array}{l}15 \\
23 \\
25 \\
30\end{array}$ & - & $\begin{array}{l}15 \\
23 \\
27 \\
30\end{array}$ & E & $\begin{array}{r}8 \\
14 \\
17 \\
20\end{array}$ & $\begin{array}{l}- \\
- \\
-\end{array}$ & $\begin{array}{l}175 \\
215 \\
235 \\
250\end{array}$ & E & $\begin{array}{l}110 \\
120 \\
130 \\
138\end{array}$ & E & $\begin{array}{l}132 \\
152 \\
165 \\
175\end{array}$ & E \\
\hline
\end{tabular}

* Studies at intermediate levels of exercise not used in diagrams or statistical analyses. 
the catheter immediately before exercise and withdrawing the tip to the main stem of the pulmonary artery at the commencement of the last minute of exercise, when pulmonary arterial pressures were recorded and mixed venous blood was withdrawn. The percentage oxygen saturation of the blood samples was estimated by a modification of the Gatman spectrophotometric technique (Wade et al., 1953) and expired gas analysis was performed by the Scholander micro-method and required to check to 0.03 per cent. The blood oxygen capacity at rest and during exercise was measured by the Van Slyke manometric and standard photometric techniques.

Throughout the results the term "systemic arterial blood pressure" is used to describe the upper body arterial pressure as determined by the intravascular brachial arterial pressure recordings. The "mean" brachial arterial pressure was calculated as the sum of the diastolic pressure and one-third of the pulse pressure.

The pulmonary arteriolar resistance and the total systemic peripheral resistance were calculated according to the methods used by Dexter et al. (1951). In patients in whom right atrial pressures were not recorded during exercise it has been assumed, for the purposes of calculating right ventricular work, that there was no rise during exercise. The pulmonary wedge pressure has been taken into account in all calculations of the left ventricular work.

The results are presented and analysed in the following form; the observations at rest, before and after operation are followed by those during exercise, also before and after operation. To simplify the statistical and diagrammatic presentation, only the data obtained during the maximum exercising period was used, although the data obtained at lesser levels of exercise is given for each patient in Table II. Control values under similar conditions were provided by studies on 16 normal subjects (Donald et al., 1955), 10 patients with normal cardiovascular systems (Donald et al., 1957) and 13 patients with uncomplicated essential hypertension (Taylor et al., 1957) previously reported from this laboratory. The important inter-relationships of a number of the hæmodynamic observations are presented in the discussion.

\section{RESULTS}

In order to clarify the presentation of these results, the mean values of the observations at rest and during exercise together with the standard error of the mean and standard deviation of observations are detailed in Tables III and IV respectively. Statistical details are only given in the text in relation to the more important findings. Description of significant or non-significant differences from normal data or between pre- and post-operative findings are based on orthodox statistical methods (Fisher, 1945).

\section{Observations At Rest Before and After Operation \\ Oxygen Uptake, Arterial Blood Oxygen Saturation, and A-V Difference}

The mean oxygen uptake at rest of the 20 untreated patients with coarctation was significantly raised $(P<0.001)$ when compared with that of normal subjects studied under similar conditions (Donald et al., 1955). The mean resting oxygen uptake of the 12 patients in whom the coarctation was resected was $163 \pm 7 \mathrm{ml} . / \mathrm{min} . / \mathrm{sq} . \mathrm{m}$. (S.D.=24) before and $145 \pm 5 \mathrm{ml} . / \mathrm{min} . / \mathrm{sq} . \mathrm{m}$. (S.D.=18) after operation, a statistically significant reduction $(0.05>P>0.02)$.

The resting systemic arterial blood oxygen saturation was normal both before and after operation. The resting arterio-venous oxygen content difference (A-V difference) was normal in all untreated patients and not significantly altered by operation.

\section{Cardiac Output, Heart Rate, Stroke Volume, and Ventilation}

The mean resting cardiac index of the 20 untreated patients was $4 \cdot 40 \pm 0 \cdot 151 . / \mathrm{min} . / \mathrm{sq} . \mathrm{m} \cdot$ $($ S.D. $=0.68)$. The mean resting cardiac index, as determined by the "Fick and catheter" method, of 111 normal subjects and patients with normal cardiovascular systems collected from the literature (Cournand et al., 1945; Stead et al., 1945; Hamilton et al., 1948; Werkö et al., 1949; Ebert et al., 1949; and Thomasson, 1957), including 21 from this laboratory (Donald et al., 1953; Donald et al., 1957), is $3.49 \pm 0.061 . / \mathrm{min} . / \mathrm{sq} . \mathrm{m}$. (S.D. $=0.67$ ). The elevation of the mean resting cardiac output in the 20 untreated patients with coarctation of the aorta is highly significant $(\mathrm{P}<0.001)$. 
TABLE III

Statistical Details of Observations at Rest

\begin{tabular}{|c|c|c|c|c|c|c|}
\hline & & \multirow{3}{*}{$\begin{array}{l}\text { Normal } \\
\text { subjects }\end{array}$} & \multicolumn{3}{|c|}{ Coarctation of aorta } & \multirow{3}{*}{$\begin{array}{c}\text { Essential } \\
\text { hypertension }\end{array}$} \\
\hline & & & \multirow{2}{*}{$\begin{array}{c}20 \text { untreated } \\
\text { patients }\end{array}$} & \multicolumn{2}{|c|}{$\begin{array}{l}12 \text { patients before } \\
\text { and after operation }\end{array}$} & \\
\hline & & & & Before & After & \\
\hline Oxygen uptake ml./min./sq.m. & - & $\begin{array}{l}139 \pm 3 \\
(12)\end{array}$ & $\begin{array}{r}164 \pm 5 \\
(22)\end{array}$ & $\begin{array}{r}163 \pm 7 \\
\text { (24) }\end{array}$ & $\begin{array}{r}145 \pm 5 \\
(18)\end{array}$ & $\begin{array}{r}146 \pm 5 \\
(17)\end{array}$ \\
\hline A-V difference, vol. per cent.. & $\cdots \quad \cdots$ & - & $\begin{array}{c}3 \cdot 71 \pm 0.09 \\
(0.40)\end{array}$ & $\begin{array}{c}3 \cdot 69 \pm 0 \cdot 12 \\
(0 \cdot 42)\end{array}$ & $\begin{array}{c}3.94 \pm 0.20 \\
(0.69)\end{array}$ & $\begin{array}{c}3 \cdot 98 \pm 0 \cdot 16 \\
(0.59)\end{array}$ \\
\hline Cardiac output, 1./min./sq.m. & . $\quad \cdots$ & $\begin{array}{c}3.49 \pm 0.06^{*} \\
(0.67)\end{array}$ & $\begin{array}{c}4 \cdot 40 \pm 0 \cdot 15 \\
(0 \cdot 68)\end{array}$ & $\begin{array}{c}4 \cdot 40 \pm 0 \cdot 22 \\
(0 \cdot 78)\end{array}$ & $\begin{array}{c}3 \cdot 80 \pm 0 \cdot 26 \\
(0 \cdot 90)\end{array}$ & $\begin{array}{c}3 \cdot 72 \pm 0.18 \\
(0.66)\end{array}$ \\
\hline Heart rate, beats/min. & $\cdots$ & $\begin{array}{r}81 \pm 3 \\
(10)\end{array}$ & $\begin{array}{c}89 \pm 3 \\
(11)\end{array}$ & $\begin{array}{c}90 \pm 4 \\
(13)\end{array}$ & $\begin{array}{c}90 \pm 5 \\
(16)\end{array}$ & $\begin{array}{r}83 \pm 3 \\
(10)\end{array}$ \\
\hline Stroke volume, ml./sq.m. & $\cdots$ & $\begin{array}{r}55 \pm 3 \\
(12)\end{array}$ & $\begin{array}{c}50 \pm 2 \\
(11)\end{array}$ & $\begin{array}{c}50 \pm 4 \\
(14)\end{array}$ & $\begin{array}{c}44 \pm 4 \\
(14)\end{array}$ & $\begin{array}{c}45 \pm 2 \\
(8)\end{array}$ \\
\hline Ventilation, $1 . / \mathrm{min} . / \mathrm{sq} . \mathrm{m}$. & $\cdots$ & $\begin{array}{c}3 \cdot 02 \pm 0 \cdot 10 \\
(0 \cdot 38)\end{array}$ & $\begin{array}{c}3 \cdot 86 \pm 0 \cdot 21 \\
(0 \cdot 95)\end{array}$ & $\begin{array}{c}3 \cdot 76 \pm 0 \cdot 21 \\
(0 \cdot 73)\end{array}$ & $\begin{array}{c}3 \cdot 69 \pm 0 \cdot 20 \\
(0 \cdot 70)\end{array}$ & $\begin{array}{c}3 \cdot 49 \pm 0.20 \\
(0.73)\end{array}$ \\
\hline Pulmonary wedge pressure (mea & $\mathrm{n}), \mathrm{mm} . \mathrm{Hg}$ & $11 \pm 0.9 \dagger$ & $\frac{10 \pm 0.7}{(3)}$ & $\frac{11 \pm 0.6}{(2)}$ & $\frac{9 \pm 0 \cdot 5}{(2)}$ & $\underset{(5)}{11 \pm 1 \cdot 4}$ \\
\hline Pulmonary arterial pressure (mea & n), mm. $\mathbf{H g}$ & $\frac{18 \pm 0.5}{(2)}$ & $\underset{(5)}{18 \pm 1 \cdot 1}$ & $\frac{19 \pm 1 \cdot 2}{(4)}$ & $\underset{(3)}{16 \pm 0.9}$ & $\underset{(7)}{20 \pm 1 \cdot 9}$ \\
\hline $\begin{array}{ccc}\text { Pulmonary } & \text { vascular } & \text { resistance, } \\
\text { cm. }{ }^{-5} . & \ldots & \cdots\end{array}$ & $\begin{array}{cc}\text { dynes } & \text { sec. } \\
\cdots & \cdots\end{array}$ & $\begin{array}{c}105 \pm 6 \\
(24)\end{array}$ & $\begin{array}{c}88 \pm 11 \\
(48)\end{array}$ & $\begin{array}{c}97 \pm 17 \\
(59)\end{array}$ & $\begin{array}{c}102 \pm 17 \\
(58)\end{array}$ & $\begin{array}{c}110 \pm 20 \\
(71)\end{array}$ \\
\hline Right ventricular work, $\mathrm{Kg} . \mathrm{m} . / \mathrm{m}$ & in./sq.m. .. & $\begin{array}{c}0.75 \pm 0.05 \\
(0.21)\end{array}$ & $\begin{array}{l}0.87 \pm 0.08 \\
(0.36)\end{array}$ & $\begin{array}{l}0.94 \pm 0.10 \\
(0.37)\end{array}$ & $\begin{array}{c}0.70 \pm 0 \cdot 05 \\
(0 \cdot 18)\end{array}$ & $\begin{array}{c}0.73 \pm 0 \cdot 12 \\
(0.43)\end{array}$ \\
\hline \multirow{3}{*}{$\begin{array}{l}\text { Systemic arterial pressure } \\
\mathrm{mm} . \mathbf{H g}\end{array}$} & Systolic & $\begin{array}{l}121 \pm 4 \\
(15)\end{array}$ & $\begin{array}{c}198 \pm 8 \\
(36)\end{array}$ & $\underset{(38)}{211 \pm 11}$ & $\begin{array}{c}156 \pm 4 \\
(14)\end{array}$ & $\begin{array}{c}205 \pm 8 \\
(28)\end{array}$ \\
\hline & Diastolic & $\begin{array}{l}71 \pm 2 \\
(10)\end{array}$ & $\begin{array}{r}107 \pm 4 \\
(18)\end{array}$ & $\begin{array}{r}114 \pm 4 \\
(13)\end{array}$ & $\frac{93 \pm 2}{(8)}$ & $\begin{array}{c}125 \pm 7 \\
(24)\end{array}$ \\
\hline & Mean & $\begin{array}{r}88 \pm 3 \\
(10)\end{array}$ & $\begin{array}{c}138 \pm 5 \\
(22)\end{array}$ & $\begin{array}{c}146 \pm 6 \\
(20)\end{array}$ & $\begin{array}{c}114 \pm 3 \\
(10)\end{array}$ & $\begin{array}{c}151 \pm 7 \\
(24)\end{array}$ \\
\hline \multicolumn{2}{|c|}{ Systemic arterial resistance, dynes sec. $\mathrm{cm} .-5$} & $\begin{array}{l}876 \pm 42 \\
\quad(168)\end{array}$ & $\begin{array}{c}1567 \pm 107 \\
(480)\end{array}$ & $\begin{array}{c}1751 \pm 151 \\
(524)\end{array}$ & $\begin{array}{c}1546 \pm 123 \\
(427)\end{array}$ & $\underset{(440)}{1911 \pm 122}$ \\
\hline \multicolumn{2}{|c|}{ Left ventricular work, Kg.m./min./sq.m. } & $\begin{array}{c}5 \cdot 11 \pm 0 \cdot 27 \\
(1 \cdot 08)\end{array}$ & $\begin{array}{c}8.09 \pm 0.41 \\
(1.83)\end{array}$ & $\begin{array}{c}8 \cdot 54 \pm 0 \cdot 54 \\
(1 \cdot 88)\end{array}$ & $\begin{array}{c}5 \cdot 82 \pm 0.48 \\
(1.68)\end{array}$ & $\begin{array}{c}7 \cdot 05 \pm 0.45 \\
(1.63)\end{array}$ \\
\hline
\end{tabular}

Mean resting values and standard error of mean with standard deviation (parenthesis) of observations in patients with coarctation of the aorta before and after operation compared with those for normal subjects (Donald et al., 1955) and patients with essential hypertension (Taylor et al., 1957) previously reported from this laboratory.

* This value was calculated from observations on 111 normal subjects collected from various papers (see text) as the cardiac output determinations in the 16 normal subjects reported by Donald et al. (1955) were before exercise and moderately raised due to excitement.

$\dagger$ This value is calculated from observations on 10 patients with normal cardiovascular systems (Donald et al. 1957). 
TABLE IV

Statistical Details of Observations During Exercise

\begin{tabular}{|c|c|c|c|c|c|c|}
\hline \multirow{3}{*}{\multicolumn{2}{|c|}{. }} & \multirow{3}{*}{$\begin{array}{l}\text { Normal } \dagger \\
\text { subjects }\end{array}$} & \multicolumn{3}{|c|}{ Coarctation of aorta } & \multirow{3}{*}{$\begin{array}{c}\text { Essential } \\
\text { hypertension }\end{array}$} \\
\hline & & & \multirow{2}{*}{$\begin{array}{c}17 \\
\text { patients } \\
\text { untreated }\end{array}$} & \multicolumn{2}{|c|}{$\begin{array}{l}12 \text { patients before } \\
\text { and after operation }\end{array}$} & \\
\hline & & & & Before & After & \\
\hline Oxygen uptake, ml./min./sq.m. & $\cdots$ & $\begin{array}{c}656 \pm 82 \\
(294)\end{array}$ & $\begin{array}{c}732 \pm 46 \\
(204)\end{array}$ & $\begin{array}{c}673 \pm 63 \\
(218)\end{array}$ & $\begin{array}{c}644 \pm 56 \\
(195)\end{array}$ & $\begin{array}{c}695 \pm 40 \\
(144)\end{array}$ \\
\hline A-V difference, vol. per cent ... & . & $\begin{array}{c}8 \cdot 53 \pm 0 \cdot 60 \\
(2 \cdot 15)\end{array}$ & $\begin{array}{c}9 \cdot 86 \pm 0 \cdot 32 \\
(1 \cdot 43)\end{array}$ & $\begin{array}{c}9 \cdot 74 \pm 0 \cdot 43 \\
(1 \cdot 49)\end{array}$ & $\begin{array}{c}9 \cdot 59 \pm 0 \cdot 51 \\
(1 \cdot 77)\end{array}$ & $\begin{array}{c}9 \cdot 97 \pm 0 \cdot 36 \\
(1 \cdot 29)\end{array}$ \\
\hline Cardiac output, 1./min./sq.m. & $\cdots$ & $\begin{array}{c}7 \cdot 37 \pm 0 \cdot 43 \\
(1 \cdot 53)\end{array}$ & $\begin{array}{c}7 \cdot 38 \pm 0 \cdot 35 \\
(1 \cdot 56)\end{array}$ & $\begin{array}{c}6.84 \pm 0.49 \\
(1.68)\end{array}$ & $\begin{array}{c}6 \cdot 68 \pm 0 \cdot 34 \\
(1 \cdot 17)\end{array}$ & $\begin{array}{c}7 \cdot 08 \pm 0 \cdot 28 \\
(1 \cdot 02)\end{array}$ \\
\hline Heart rate, beats/min. & . & $\begin{array}{c}122 \pm 8 \\
(27)\end{array}$ & $\begin{array}{c}151 \pm 4 \\
(19)\end{array}$ & $\begin{array}{c}147 \pm 6 \\
(20)\end{array}$ & $\begin{array}{c}144 \pm 7 \\
(24)\end{array}$ & $\begin{array}{c}138 \pm 4 \\
(14)\end{array}$ \\
\hline Stroke volume, ml./sq.m. & $\cdots$ & $\begin{array}{c}61 \pm 2 \\
(8)\end{array}$ & $\begin{array}{c}49 \pm 3 \\
(11)\end{array}$ & $\begin{array}{c}47 \pm 3 \\
(11)\end{array}$ & $\frac{47 \pm 2}{(8)}$ & $\begin{array}{c}51 \pm 2 \\
(7)\end{array}$ \\
\hline Ventilation, $1 . / \mathrm{min} . / \mathrm{sq} . \mathrm{m}$. & . & $\begin{array}{c}11 \cdot 80 \pm 1 \cdot 65 \\
(5 \cdot 95)\end{array}$ & $\begin{array}{c}15 \cdot 19 \pm 1 \cdot 02 \\
(4 \cdot 57)\end{array}$ & $\begin{array}{c}14 \cdot 32 \pm 1 \cdot 50 \\
(5 \cdot 20)\end{array}$ & $\begin{array}{c}13 \cdot 37 \pm 1 \cdot 32 \\
(4 \cdot 58)\end{array}$ & $\begin{array}{c}16 \cdot 63 \pm 1 \cdot 78 \\
(6 \cdot 42)\end{array}$ \\
\hline Pulmonary wedge pressure (mea & n), $\mathrm{mm} . \mathbf{H g}$ & $\frac{14 \pm 3^{*}}{(7)}$ & $\begin{array}{c}18 \pm 2 \\
(8)\end{array}$ & $\begin{array}{c}18 \pm 2 \\
(7)\end{array}$ & $\stackrel{14 \pm 1}{(4)}$ & $\begin{array}{r}23 \pm 6 \\
(12)\end{array}$ \\
\hline Pulmonary arterial pressure (me & an), $\mathrm{mm} . \mathbf{H g}$ & $\begin{array}{c}27 \pm 3 \\
(9)\end{array}$ & $\begin{array}{l}29 \pm 2 \\
(11)\end{array}$ & $\begin{array}{r}29 \pm 3 \\
(10)\end{array}$ & $\begin{array}{c}27 \pm 2 \\
(8)\end{array}$ & $\begin{array}{r}34 \pm 3 \\
(10)\end{array}$ \\
\hline $\begin{array}{cccc}\text { Pulmonary } & \text { vascular } & \text { resistance, } \\
\text { cm. } & -5 \\
& & \ldots & \ldots\end{array}$ & $\begin{array}{cc}\text { dynes } & \text { sec. } \\
\ldots & \cdots\end{array}$ & $\begin{array}{c}105 \pm 10 \\
(36)\end{array}$ & $\begin{array}{c}75 \pm 12 \\
(54)\end{array}$ & $\begin{array}{c}89 \pm 18 \\
(63)\end{array}$ & $\underset{(65)}{101 \pm 19}$ & $\begin{array}{c}92 \pm 27 \\
(97)\end{array}$ \\
\hline Right ventricular work, Kg.m & ./min./sq.m. & $\begin{array}{c}2 \cdot 18 \pm 0 \cdot 45 \\
(1 \cdot 62)\end{array}$ & $\begin{array}{c}2 \cdot 42 \pm 0 \cdot 24 \\
(1 \cdot 05)\end{array}$ & $\begin{array}{c}2 \cdot 27 \pm 0 \cdot 26 \\
(0 \cdot 91)\end{array}$ & $\begin{array}{c}2 \cdot 14 \pm 0 \cdot 24 \\
(0 \cdot 83)\end{array}$ & $\begin{array}{c}2 \cdot 63 \pm 0.20 \\
(0.73)\end{array}$ \\
\hline \multirow{3}{*}{$\begin{array}{l}\text { Systemic arterial pressure } \\
\mathrm{mm} . \mathbf{H g}\end{array}$} & Systolic & $\begin{array}{c}165 \pm 8 \\
(26)\end{array}$ & $\begin{array}{c}266 \pm 10 \\
(47)\end{array}$ & $\begin{array}{c}266 \pm 15 \\
(52)\end{array}$ & $\begin{array}{c}206 \pm 9 \\
(32)\end{array}$ & $\begin{array}{c}246 \pm 13 \\
(47)\end{array}$ \\
\hline & Diastolic & $\begin{array}{c}87 \pm 4 \\
(14)\end{array}$ & $\begin{array}{c}129 \pm 5 \\
(21)\end{array}$ & $\begin{array}{c}134 \pm 6 \\
(19)\end{array}$ & $\begin{array}{c}109 \pm 5 \\
(17)\end{array}$ & $\begin{array}{c}146 \pm 8 \\
(27)\end{array}$ \\
\hline & Mean & $\begin{array}{r}107 \pm 4 \\
(14)\end{array}$ & $\begin{array}{c}175 \pm 6 \\
(25)\end{array}$ & $\begin{array}{c}178 \pm 8 \\
(28)\end{array}$ & $\begin{array}{c}141 \pm 6 \\
(20)\end{array}$ & $\begin{array}{c}180 \pm 9 \\
(32)\end{array}$ \\
\hline \multicolumn{2}{|c|}{ Systemic arterial resistance, dynes sec. $\mathrm{cm} .^{-5}$} & $\begin{array}{c}650 \pm 45 \\
(51)\end{array}$ & $\begin{array}{c}1225 \pm 95 \\
(423)\end{array}$ & $\begin{array}{c}1406 \pm 146 \\
(506)\end{array}$ & $\begin{array}{l}1057 \pm 63 \\
\quad(219)\end{array}$ & $\begin{array}{l}1200 \pm 68 \\
(246)\end{array}$ \\
\hline \multicolumn{2}{|c|}{ Left ventricular work, Kg.m./min./sq.m. } & $\begin{array}{c}10 \cdot 29 \pm 1 \cdot 14 \\
(3 \cdot 78)\end{array}$ & $\begin{array}{c}16 \cdot 66 \pm 0 \cdot 94 \\
(4 \cdot 21)\end{array}$ & $\begin{array}{c}15 \cdot 93 \pm 1 \cdot 46 \\
(5 \cdot 05)\end{array}$ & $\begin{array}{c}12 \cdot 43 \pm 1 \cdot 03 \\
(3 \cdot 57)\end{array}$ & $\begin{array}{c}15 \cdot 32 \pm 1 \cdot 12 \\
(4 \cdot 03)\end{array}$ \\
\hline
\end{tabular}

Mean values during exercise and standard error of mean with standard deviation (parenthesis) of observations in patients with coarctation of the aorta before and after operation compared with those for normal subjects (Donald et al., 1955) and patients with essential hypertension (Taylor et al., 1957) previously reported from this laboratory.

* This value is calculated from observations on 7 patients with normal cardiovascular systems (Donald et al., 1957).

$\dagger$ These values were calculated from observations on 13 of 16 normal subjects who were exercising at similar levels of oxygen uptake (Donald et al., 1955). 
In 6 of these 20, the resting cardiac output was more than two standard deviations ( $>4.831 . / \mathrm{min}$./ sq.m.) greater than the "normal" mean (Fig. 1). The mean cardiac output at rest before operation of the 12 patients in whom the coarctation was resected was identical with that of the whole untreated group. After resection of the coarctation there was a small but statistically insignificant reduction of resting mean cardiac output from 4.40 before to $3.801 . / \mathrm{min}$./sq.m. after operation.

When studied individually, the cardiac output was reduced after operation in six, remained unchanged in five, and increased in one (Fig. 1). In the six patients in whom a reduction of cardiac output was observed, it was considerable, being from a mean value of 4.31 before to $3.031 . / \mathrm{min}$./ sq.m. after operation. It is unlikely that the greater excitement or emotional upset were responsible for the raised cardiac output observed during the initial investigation as both the mean heart rate and mean ventilatory volumes were normal and not significantly different on either occasion.

The mean resting heart rate of the whole group of 20 untreated patients was 89 beats a minute. The mean heart rate of the 12 patients in whom the coarctation was resected was nearly identical and was unchanged after operation.

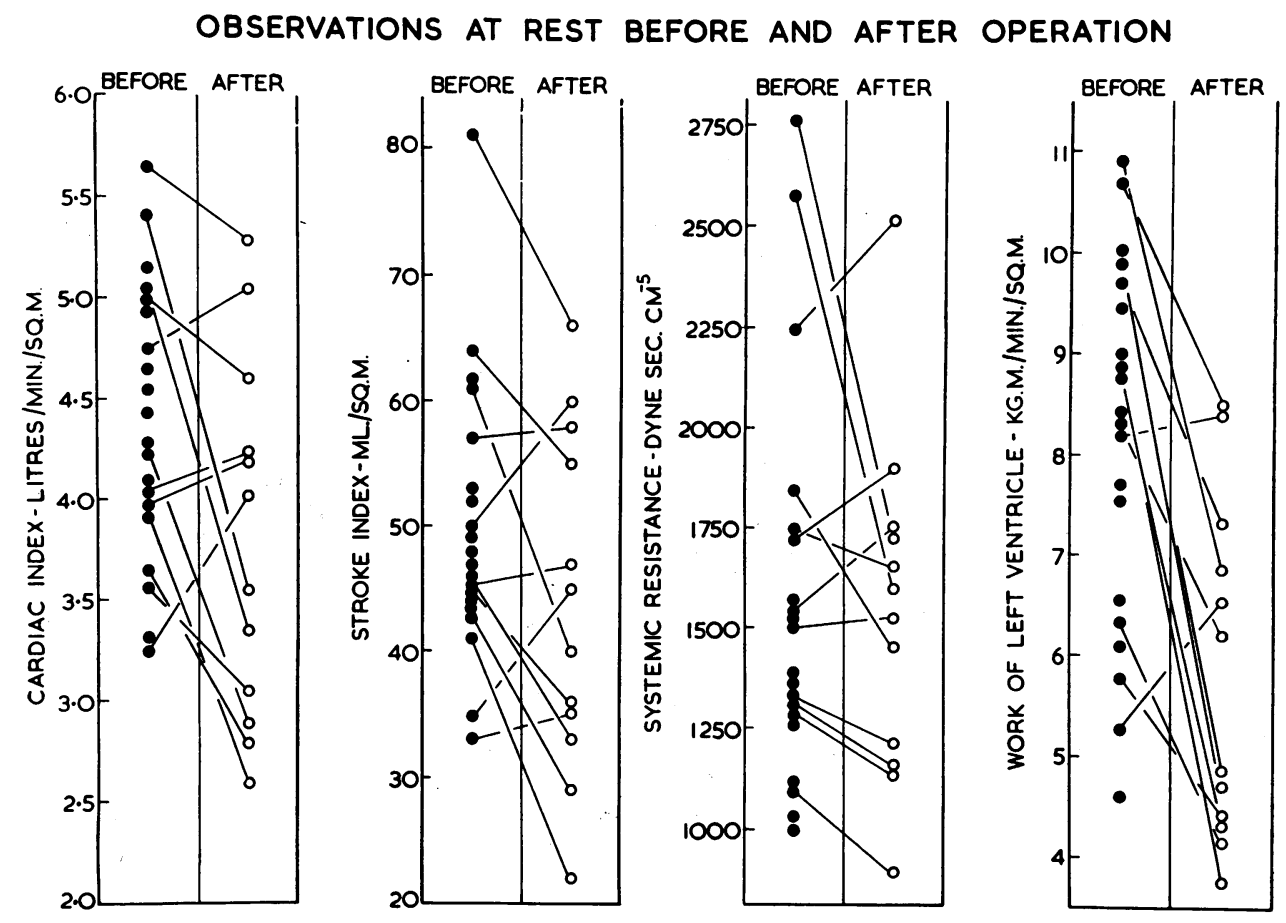

Fig. 1.-The cardiac and stroke indices, systemic vascular resistance, and work of the left ventricle at rest in 20 patients before and in 12 patients after operation also.

The mean stroke volume of the whole untreated group was $50 \mathrm{ml} . / \mathrm{sq} . \mathrm{m}$. The mean preoperative stroke volume of the 12 patients who underwent operation was almost identical and there was a small but non-significant reduction after operation (Fig. 1).

The resting ventilation of all the untreated patients were normal (mean $3.861 . / \mathrm{min} . / \mathrm{sq} . \mathrm{m}$.). The resting ventilatory volumes of those undergoing operation were normal and almost identical with these values both before and after operation.

\section{Vascular Pressures, Resistances, and Ventricular Work}

The resting mean pulmonary wedge pressure was normal in the whole of the untreated group (mean, $10 \mathrm{~mm}$. Hg, range 4-15) and was essentially unchanged after operation (Fig. 2). 

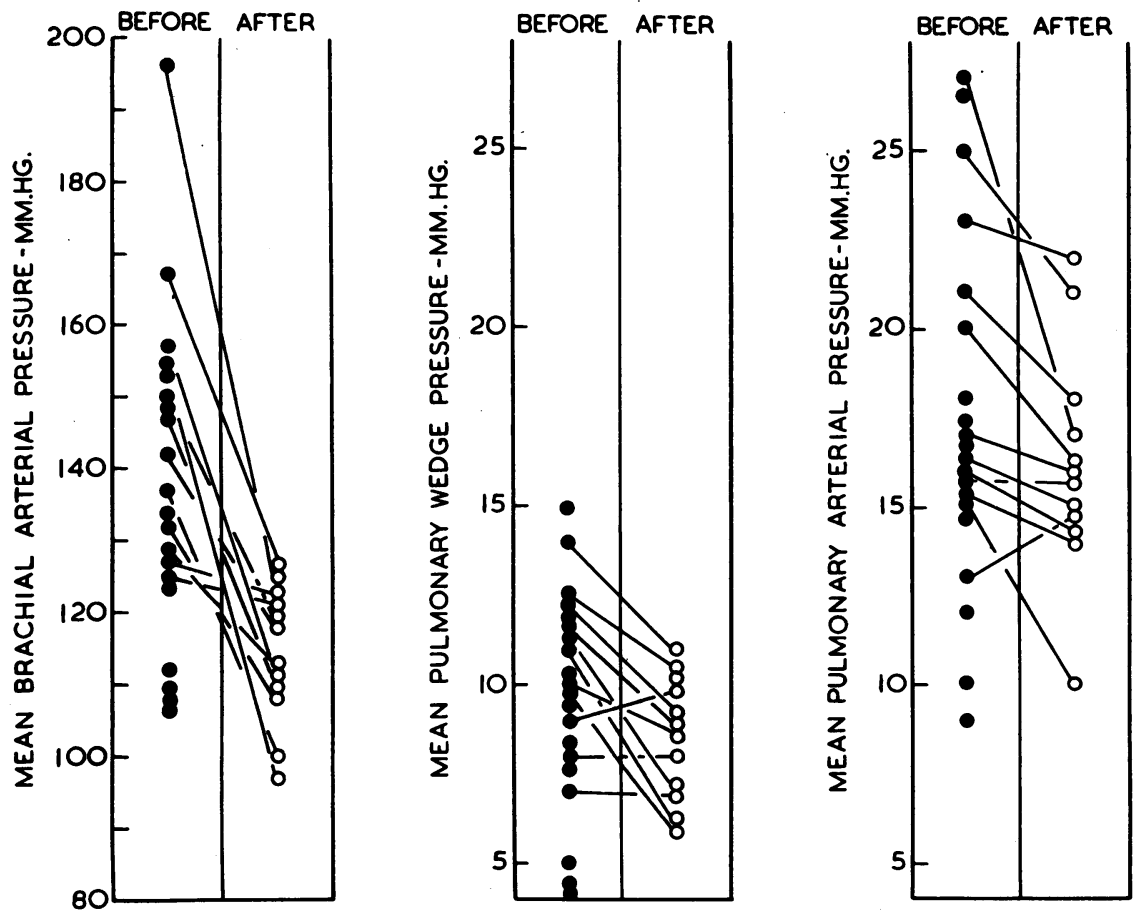

FIG. 2.-Vascular pressures at rest before and after operation. The mean brachial arterial, pulmonary wedge, and pulmonary arterial pressure at rest in 20 patients before and in 12 after operation also.

The mean pulmonary arterial pressure at rest before operation was normal in all but three instances (Fig. 2). The upper limit of normal resting pulmonary arterial pressure in this laboratory is $23 \mathrm{~mm}$. Hg. The 12 patients in whom the coarctation was resected included the two (CN17 and CN19) with mild pulmonary hypertension (27 and $25 \mathrm{~mm}$. $\mathrm{Hg}$ respectively). In these two the pressures reverted to high normal values $(18$ and $21 \mathrm{~mm}$. $\mathrm{Hg}$ respectively) after operation. The remaining patients showed a small but statistically insignificant reduction of the resting pulmonary arterial pressure after operation.

The pulmonary vascular resistance at rest was normal in all untreated patients (mean 88 dynes sec. $\mathrm{cm}^{-5}$ ). In the 12 undergoing operation it was likewise normal and unchanged by operation.

The work of the right ventricle against pressure at rest was within normal limits in all the untreated patients. In the 12 in whom the coarctation was resected, the mean right ventricular work was reduced from 0.94 before to $0.70 \mathrm{Kg} . \mathrm{m} . / \mathrm{min}$./sq.m. after operation. Although this difference of the mean values is not statistically significant, the right ventricular work was reduced in all but one of the 12 patients following operation and in four it was reduced by more than 30 per cent of the pre-operative value. This fall was due to a slight reduction of both pulmonary arterial pressure and blood flow.

The brachial arterial blood pressure at rest before and after operation is shown related to age in Fig. 3, together with the regression line and 95 per cent confidence limits for normal subjects established by Hamilton et al. (1954).

All untreated patients had an abnormal elevation of the brachial systolic pressure which was accompanied by diastolic hypertension in all but four. Before operation, at rest, the mean systolic pressure of the whole group was $198 \pm 8 \mathrm{~mm}$. $\mathrm{Hg}$ (S.D.=36), the mean diastolic pressure was $107 \pm 4 \mathrm{~mm}$. (S.D. $=18)$ and the mean mean arterial pressure was $138 \pm 5 \mathrm{~mm}$. (S.D.=22). The 
corresponding pre-operative values for the 12 patients in whom the coarctation was later resected were $211 \pm 11 \mathrm{~mm}$. (S.D.=38), $114 \pm 4 \mathrm{~mm}$. (S.D.=13), and $146 \pm 6 \mathrm{~mm}$. Hg (S.D.=20), values not significantly different from those of the group as a whole.

Following operation in these 12, the mean systolic pressure at rest was $156 \pm 4 \mathrm{~mm} . \mathrm{Hg}$ (S.D.=14), the mean diastolic pressure was $93 \pm 2 \mathrm{~mm}$. (S.D. $=8$ ), and the mean of the mean arterial pressure was $114 \pm 3 \mathrm{~mm}$. (S.D.=10). These reductions in the mean systolic, diastolic, and mean arterial pressures at rest of $55 \mathrm{~mm}$. (range 0-140), $21 \mathrm{~mm}$. (range 0-44), and $32 \mathrm{~mm}$. (range 2-73)

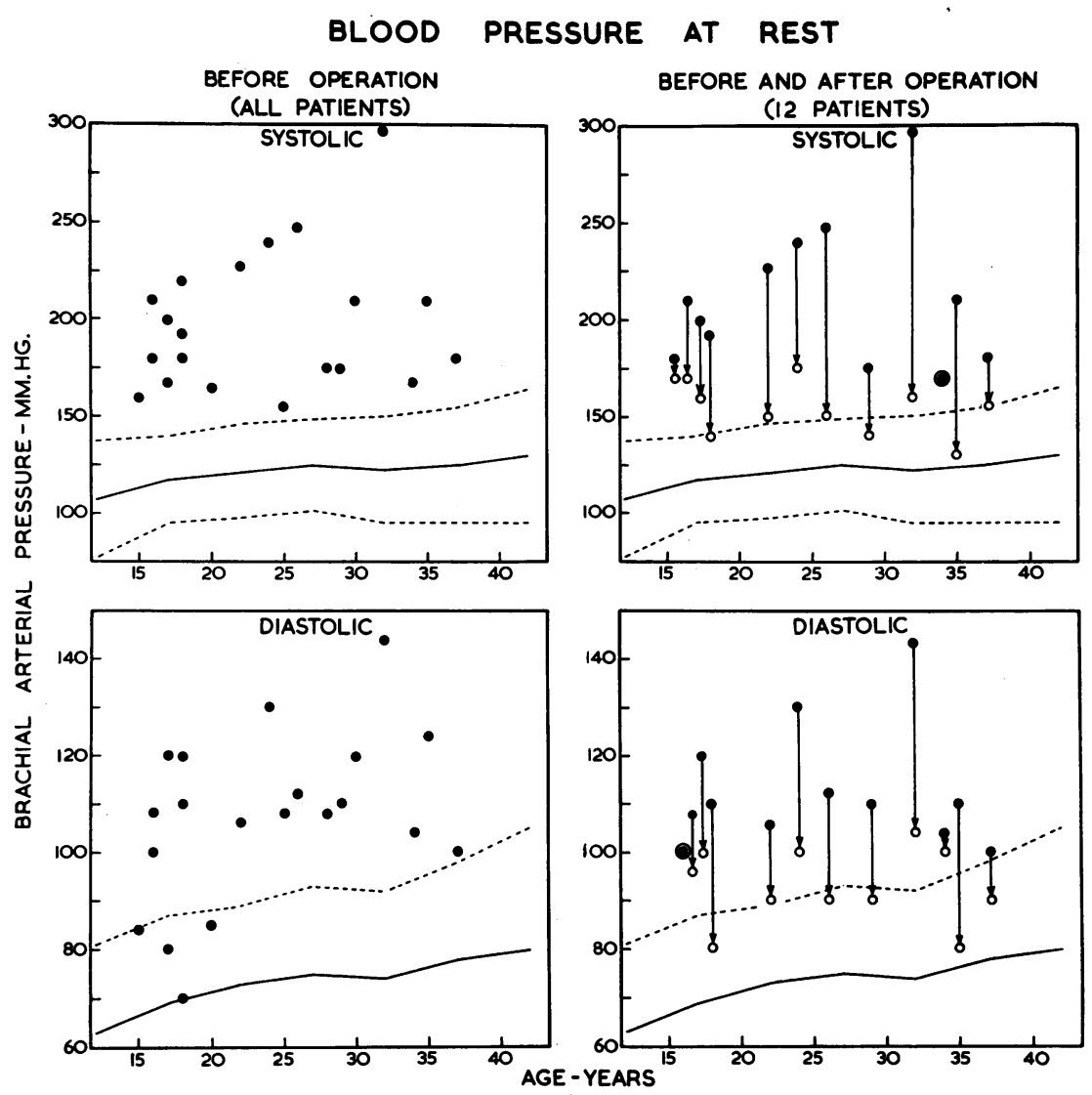

FIG. 3. - The resting brachial arterial systolic and diastolic pressures related to age, in 20 patients before and in 12 after operation also. The mean values, together with two standard deviations, of a sample of the normal population of the same age range are also given (Hamilton et al., 1954).

respectively, are all highly significant $(\mathrm{P}<0.001)$. Ten of the 12 studied showed an appreciable fall in both systolic and diastolic brachial arterial pressures after operation and in six of these ten the resting systemic arterial pressures had returned to "normal" levels (Fig. 2 and 3).

The tendency of the blood pressure to fall after operation did not appear to be related to age. The two patients in whom no reduction was achieved by operation were 16 and 34 years respectively and the greatest single reduction was observed in a patient 32 years of age. The degree of reduction of the brachial arterial pressure after operation was approximately directly proportional to its initial height. These findings are in agreement with those of Counihan (1956).

The mean systemic vascular resistance at rest of the 20 patients before operation was 1567 dynes sec. $\mathrm{cm} .{ }^{-5}$. This is significantly greater $(\mathrm{P}<0.001)$ than the corresponding value of 876 dynes sec. 
cm. ${ }^{-5}$ in normal subjects (Donald et al., 1955) but significantly less $(0.05>P>0.02)$ than the value of 1911 dynes sec. $\mathrm{cm}^{-5}$ reported in patients with uncomplicated essential hypertension (Taylor et al., 1957).

The mean systemic resistance at rest before operation in the 12 patients in whom the coarctation was later resected was $1751 \pm 151$ dynes sec. $\mathrm{cm} .{ }^{-5}($ S.D. $=524)$, a value not significantly different from the group as a whole. After operation the mean resting systemic resistance of these patients was $1546 \pm 123$ dynes sec. $\mathrm{cm} .^{-5}$ (S.D. $=427$ ), a value less but not significantly different from that before operation. In two $(\mathrm{CN} 1$ and $\mathrm{CN} 3)$ the resting systemic resistance after operation was reduced in each by 38 per cent of the pre-operative value (Fig. 1). In six there were much smaller post-operative reductions, in three there were small increases, and in one the systemic resistance was unchanged.

The mean work of the left ventricle against pressure at rest in the 20 untreated patients was $8.09 \mathrm{Kg} . \mathrm{m} . / \mathrm{min} . / \mathrm{sq} . \mathrm{m}$. This is significantly greater than the mean value of $5.11 \mathrm{Kg} . \mathrm{m} . / \mathrm{min} . / \mathrm{sq} . \mathrm{m}$. reported in normal subjects $(\mathrm{P}<0 \cdot 001)$. The mean resting work of the left ventricle against pressure in the 12 patients in whom the coarctation was later resected was $8.54 \mathrm{Kg} . \mathrm{m} . / \mathrm{min} . / \mathrm{sq} . \mathrm{m}$. before and $5.82 \mathrm{Kg} . \mathrm{m} . / \mathrm{min} . / \mathrm{sq} . \mathrm{m}$. after operation. This reduction in the mean left ventricular work after operation is highly significant $(0.01>P>0.001)$. The individual values before and after operation of the 12 in whom the coarctation was resected are shown in Fig. 1. One (CN4) showed no change and another (CN2) a slight increase in the resting left ventricular work. In the remaining ten the post-operative reduction in left ventricular work was considerable; in four the reduction was greater than 50 per cent of the pre-operative value and in the remaining six it was greater than 20 per cent. It is of interest that, as the systemic vascular resistance of many of the operated cases did not fall greatly (see Fig. 1 and 11), the considerable reduction of left ventricular work must be mainly attributed to the reduction in resting cardiac output.

\section{ObServations During Exercise Before AND After Operation}

\section{Oxygen Uptake, Arterial Blood Oxygen Saturation, and A-V Difference}

The oxygen uptake during supine leg exercise in the group of 20 untreated patients ranged from 315 to $1106 \mathrm{ml} . / \mathrm{min} . / \mathrm{sq} . \mathrm{m}$. with a mean value of $732 \mathrm{ml} . / \mathrm{min} . / \mathrm{sq} . \mathrm{m}$. (Table IV). In the 12 who underwent operation the range and mean value of exercising oxygen uptakes were of the same order both before and after operation. The differences in the oxygen uptake during exercise before and after operation in individual patients ranged from 6 to $106 \mathrm{ml} . / \mathrm{min} . / \mathrm{sq} . \mathrm{m}$. with a mean difference of $50 \mathrm{ml} . / \mathrm{min} . / \mathrm{sq} . \mathrm{m}$. and a mean percentage difference of 9 per cent of the pre-operative value.

The arterial blood oxygen saturation during exercise was normal in all patients, both before and after operation.

The exercising $A-V$ difference is related to the oxygen uptake in Fig. 4. The values of all untreated patients are shown together with those found in 12 before and after operation. The regression line and 95 per cent confidence limits of the values found in normal subjects during exercise are also given. Four of the untreated patients had abnormally high exercising A-V differences. All these four underwent operation: the exercising A-V difference returned to normal in two but was unchanged in the other two. The mean exercising A-V difference in the untreated group and in the patients undergoing resection either before or after operation, were not significantly different.

\section{Cardiac Output, Heart Rate, Stroke Volume, and Ventilation}

The cardiac output on exercise of all patients studied before and after operation is shown in Fig. 4 together with the regression line and 95 per cent confidence limits found in normal subjects. Four (CN1, CN7, CN8, and CN10) showed an exercising cardiac output just below the normal 
95 per cent confidence limits and in all four the exercising pulmonary wedge pressure was raised $(20,31,26$, and $15 \mathrm{~mm}$. $\mathrm{Hg}$ respectively).

After aortic reconstruction the cardiac output response to exercise was largely unchanged in those patients in whom it was initially normal. In the four in whom the exercising cardiac outputs response was previously slightly subnormal, operation was associated with a return to a normal exercising response in two (CN1, and CN10). In the other two (CN7 and CN8) the exercising cardiac output was still slightly subnormal 11 and 18 months after operation. The exercising wedge pressures of all these four patients returned to normal after operation. The mean cardiac outputs during exercise of the untreated group and of those undergoing resection, either before or after operation, were not significantly different.
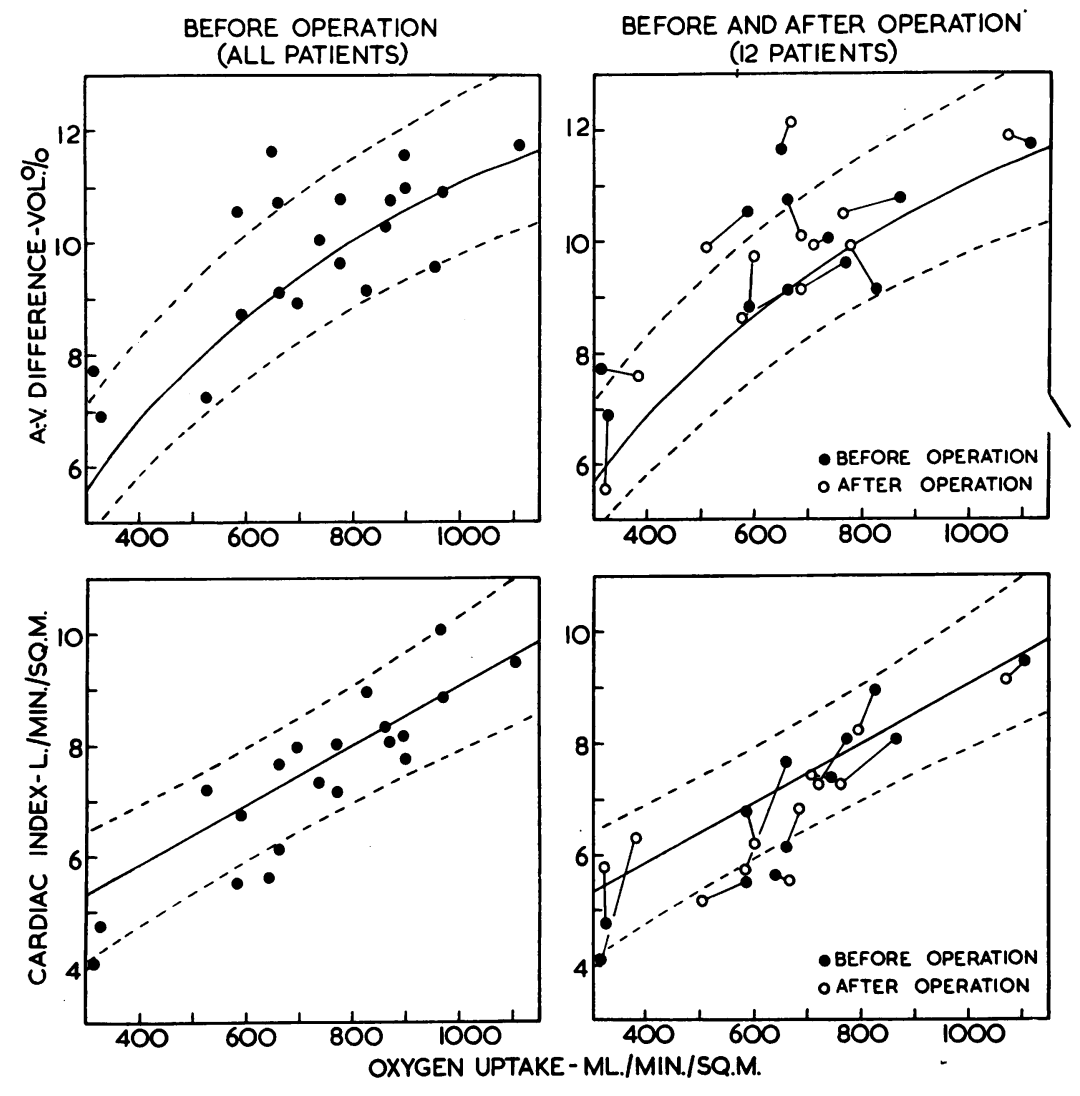

FIG. 4.-The A-V differences and cardiac indices during exercise related to oxygen uptake in 20 patients before and in 12 after operation also. The regression line and 95 per cent confidence limits for normal subjects of the same age range are also given (Donald et al., 1955).

The heart rate during exercise was largely unchanged after operation although 3 of the 12 patients showed a decrease of more than 20 beats a minute at the same level of exercise (Fig. 5). The mean values of heart rate during exercise at similar levels of oxygen uptake before and after operation were not, however, significantly different.

The exercising stroke volume was exceedingly variable in the untreated group and in the patients 
undergoing resection, both before and after operation (Fig. 5). Operation was not associated with any change in the mean exercising stroke index.

The pulmonary ventilation on exercise is related to the oxygen uptake in Fig. 6 . In the untreated group all but four patients had a normal ventilatory response to exercise: these four with hyperventilation (CN2, CN7, CN12, and $\mathrm{CN} 14)$ all had raised pulmonary wedge pressures on exercise $(18,31,17$, and $21 \mathrm{~mm}$. Hg respectively). However, seven others who had raised exercising pulmonary wedge pressures had a normal ventilatory response. (As in mitral stenosis the exercising ventilation does not appear to be a function of the exercising wedge pressure).

In those who underwent operation there was no significant overall change in ventilatory response to exercise, despite much reduction of exercising wedge pressures in many instances. The ventilatory response to exercise after operation of the four in whom it was increased before operation was reduced to normal limits in two $(\mathrm{CN} 2$ and $\mathrm{CN} 7)$ but was still slightly raised in the other two.

HEART RATE AND STROKE VOLUME DURING EXERCISE
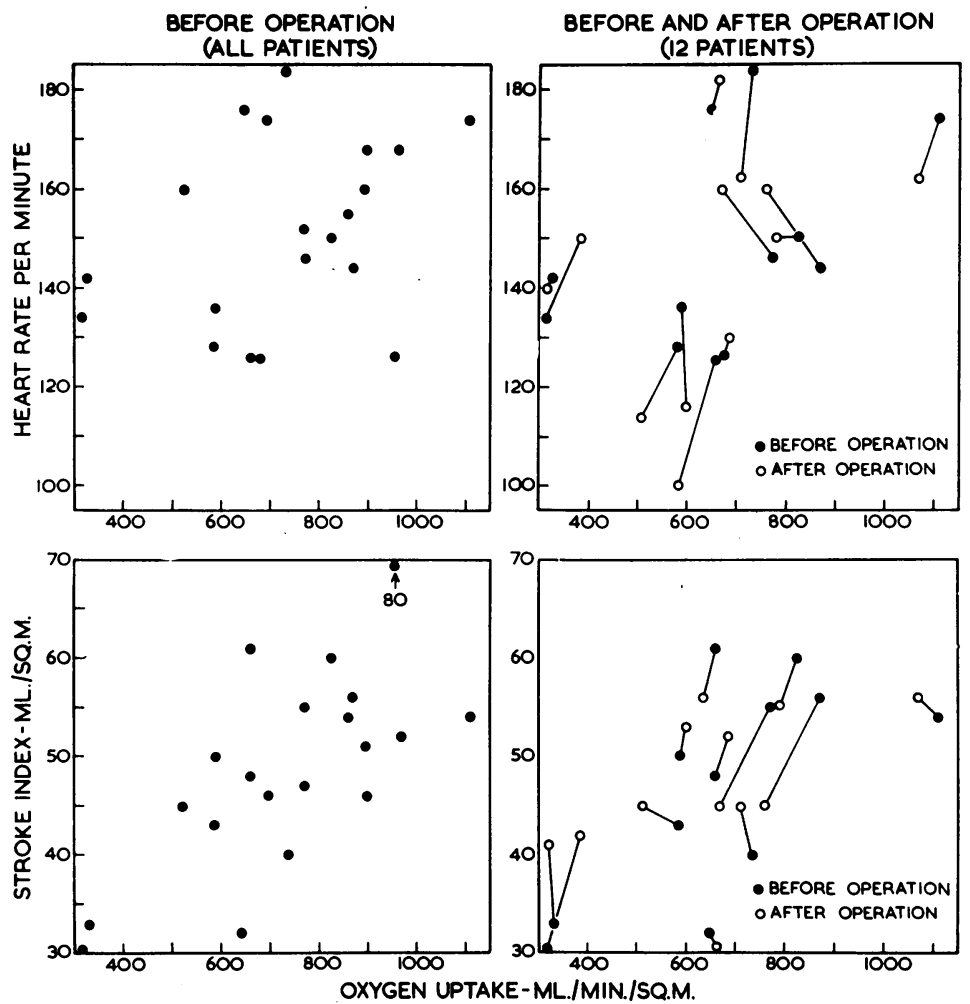

FIG. 5.-The theart rates and stroke indices during exercise related to oxygen uptake in 20 patients before and in 12 patients after operation as well.

Vascular Pressures, Resistances, and Ventricular Work

The mean pulmonary wedge pressure during exercise is related to the oxygen uptake in Fig. 7. In this laboratory the highest wedge pressure found in normal subjects of the same age group at any level of exercise is $15 \mathrm{~mm}$. $\mathrm{Hg}$ (mean). The exercising wedge pressure was abnormally raised in 13 of the 20 untreated patients, four of whom also had a slightly reduced cardiac output response to exercise. In nine, therefore, the only objective sign of left ventricular insufficiency was the raised pulmonary wedge pressure during exercise. Eight with raised exercising wedge pressures were re-studied after operation and there was a return to normal levels of exercising wedge pressure at 
similar degrees of exertion in seven instances. In the remaining one (CN3), although the mean exercising arterial blood pressure fell from 237 to $161 \mathrm{~mm}$. $\mathrm{Hg}$ the wedge pressure remained at a high level $(26 \mathrm{~mm}$. $\mathrm{Hg})$.

The mean pulmonary arterial pressure during exercise in the untreated patients and in those after operation are related to oxygen uptake in Fig. 7. The highest pulmonary arterial pressure encountered in exercising healthy subjects of the same age group is $32 \mathrm{~mm} . \mathrm{Hg}$. Before operation three (CN7, CN18, and CN19) had pulmonary hypertension on exercise. All three had normal wedge pressures at rest but showed a marked rise of wedge pressures during exercise (to 31,28 , and $36 \mathrm{~mm}$. $\mathrm{Hg}$ respectively).

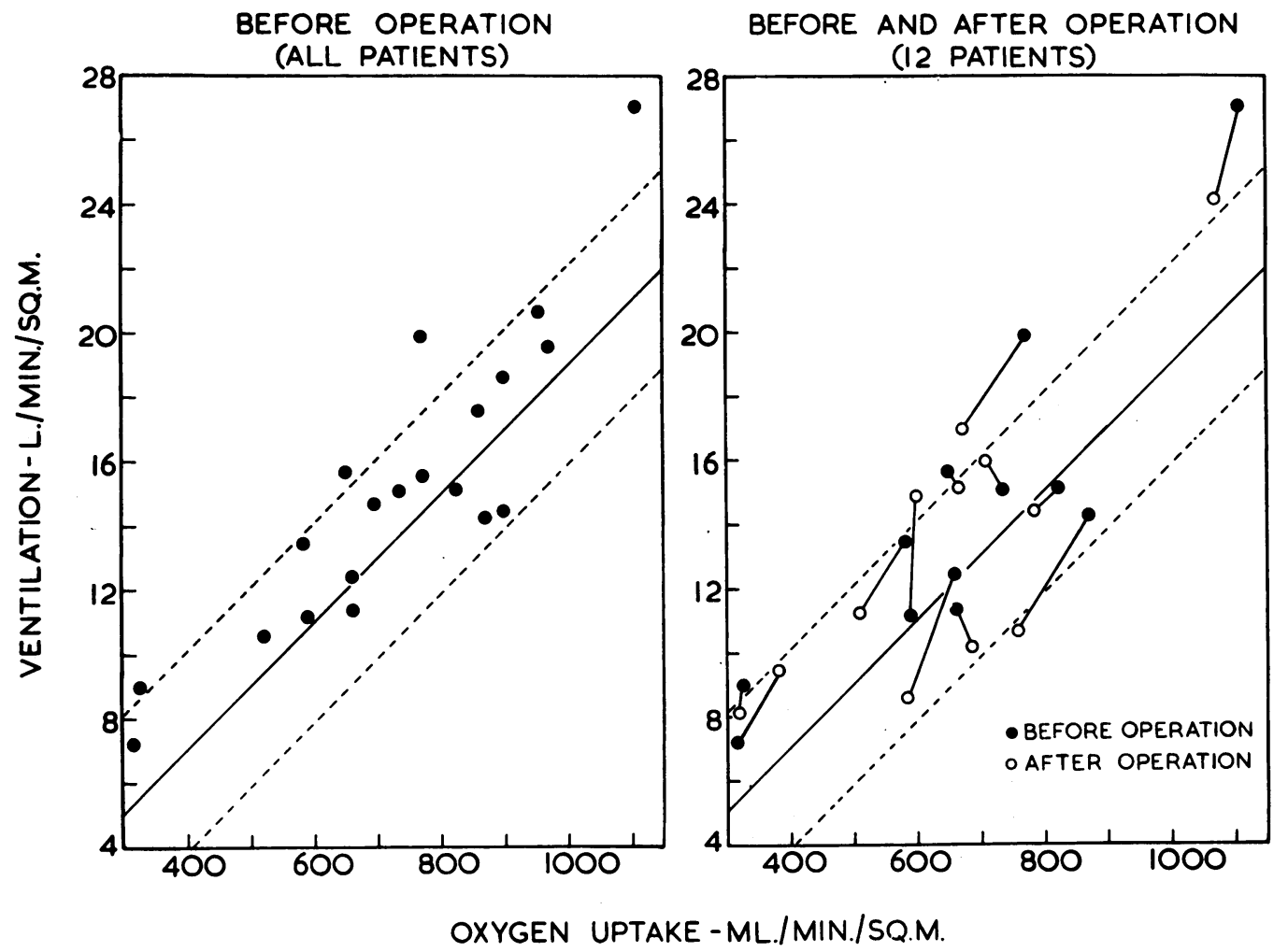

FIG. 6.-The ventilation during exercise related to oxygen uptake in 20 patients before and in 12 after operation also. The regression line and 95 per cent confidence limits for normal subjects are also given (Donald et al., 1955).

It is of interest that in two patients with high normal pre-operative pulmonary arterial pressures, the pressure was slightly higher after operation despite a fall in wedge pressure $(\mathrm{CN} 1, \mathrm{CN} 3, \mathrm{CN} 8$, and CN17). It is possible that the reduction of the left atrial pressure, as judged by the wedge pressure, reduced the degree of distension of the pulmonary vascular tree and increased the vascular resistance slightly.

The pulmonary vascular resistance during exercise of the untreated patients was not significantly different from that of normal subjects at similar levels of exertion. In the 12 who underwent operation the mean pulmonary vascular resistance was normal before and increased slightly, but not significantly, after operation.

The systemic brachial arterial blood pressure at rest and during exercise in all 20 patients, before operation, is illustrated in Fig. 8. The most notable feature is the large resting pulse pressure in the upper part of the body which is greatly increased during exercise in the majority. Exertion at any but very moderate levels frequently produced a large rise in diastolic pressure and an even 
greater rise in systolic pressure. At a mean exercising oxygen uptake of $732 \pm 46 \mathrm{ml} / \mathrm{min} . / \mathrm{sq} . \mathrm{m}$. $($ S.D. $=204)$ the mean systolic pressure of the whole group was $266 \pm 10 \mathrm{~mm}$. (S.D. $=47$ ), the mean diastolic pressure was $129 \pm 5 \mathrm{~mm}$. (S.D. $=21)$ and the mean exercising mean arterial pressure was $175 \pm 6 \mathrm{~mm}$. $\mathrm{Hg}$ (S.D. $=25)$.

The corresponding pre-operative exercising values for the 12 in whom the coarctation was later resected were $266 \pm 15$ (S.D. $=52), 134 \pm 6$ (S.D.=19), and $178 \pm 8 \mathrm{~mm}$. (S.D.=28) at a mean exercising oxygen uptake of $673 \pm 63 \mathrm{ml} . / \mathrm{min} . / \mathrm{sq} . \mathrm{m}$. (S.D.=218). These pressures and the oxygen uptake are not significantly different from those of the untreated group as a whole. Following operation in these 12, at a mean exercising oxygen uptake of $644 \pm 56 \mathrm{ml} . / \mathrm{min} . / \mathrm{sq} . \mathrm{m}$. (S.D. $=195$ ), the mean systolic pressure was $206 \pm 9 \mathrm{~mm}$. (S.D. $=32$ ), the mean diastolic pressure was $109 \pm 5 \mathrm{~mm}$. (S.D. $=17)$, and the mean of the mean arterial pressure was $141 \pm 6 \mathrm{~mm}$. $\mathrm{Hg}($ S.D. $=20)$. These reductions at approximately the same levels of exercise in the mean systolic, diastolic, and mean brachial arterial pressures after operation of 60 (range 0-120), 23 (range 10-54), and $37 \mathrm{~mm}$. $\mathrm{Hg}$ (range 6-76) are all highly significant $(0.01>\mathrm{P}>0.001)$. The individual response of the brachial arterial pressure during exercise compared with the resting level before and after operation is shown Fig. 9. In 10 of the 12 in whom the coarctation was resected, there was an appreciable reduction in the resting systolic and diastolic pressures after operation with a narrowing of the pulse pressure in the majority. After operation the response of the brachial arterial pressure to exercise is modified in that the absolute pressures are considerably reduced, although still abnormal in the majority of instances.

\section{PULMONARY PRESSURES DURING EXERCISE}
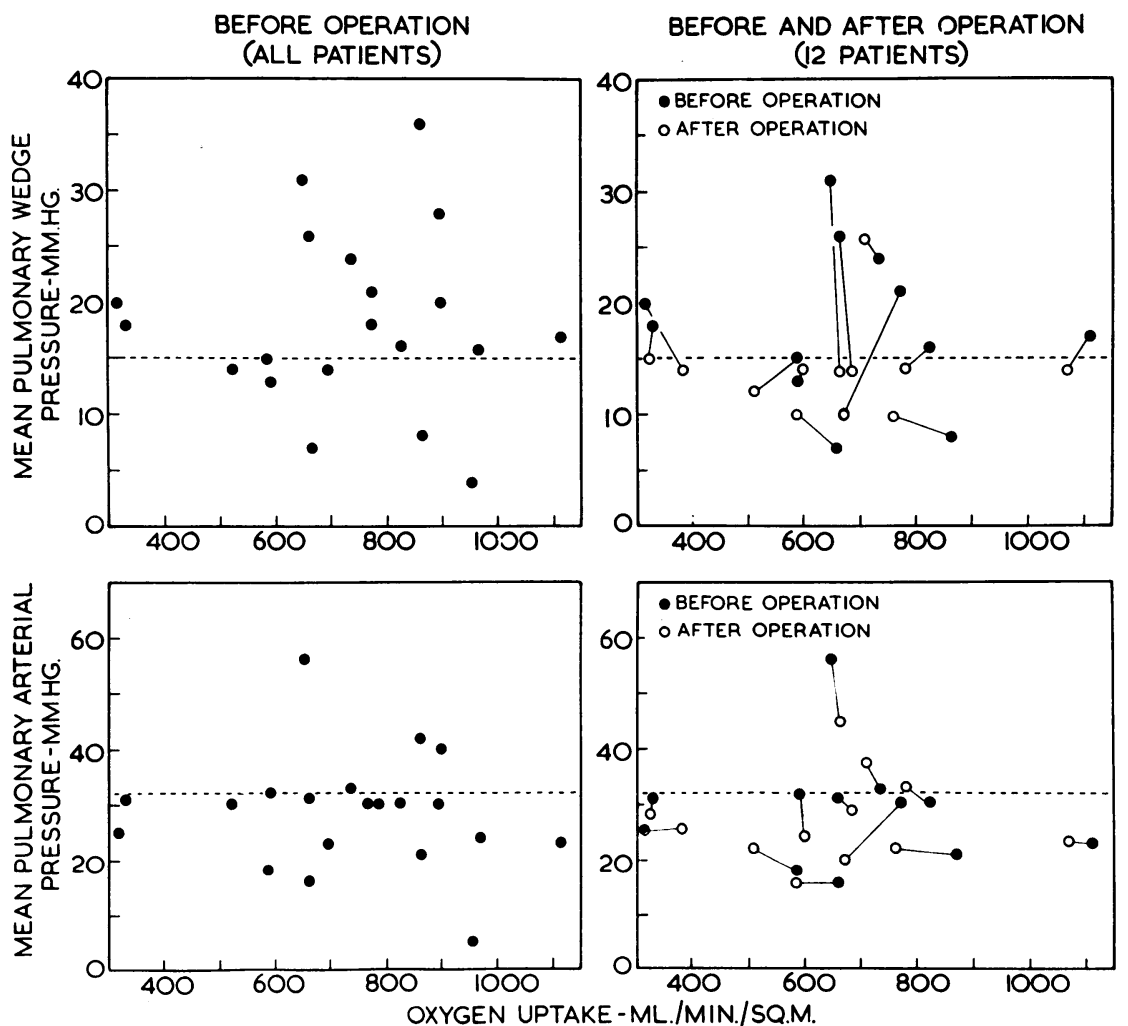

FIG. 7.-The mean pulmonary wedge and pulmonary arterial pressures during exercise related to oxygen uptake in 20 patients before and in 12 after operation also. The upper limit of normal exercising pressures observed in this laboratory are also given. 


\section{BLOOD PRESSURE AT REST AND DURING EXERCISE BEFORE OPERATION}

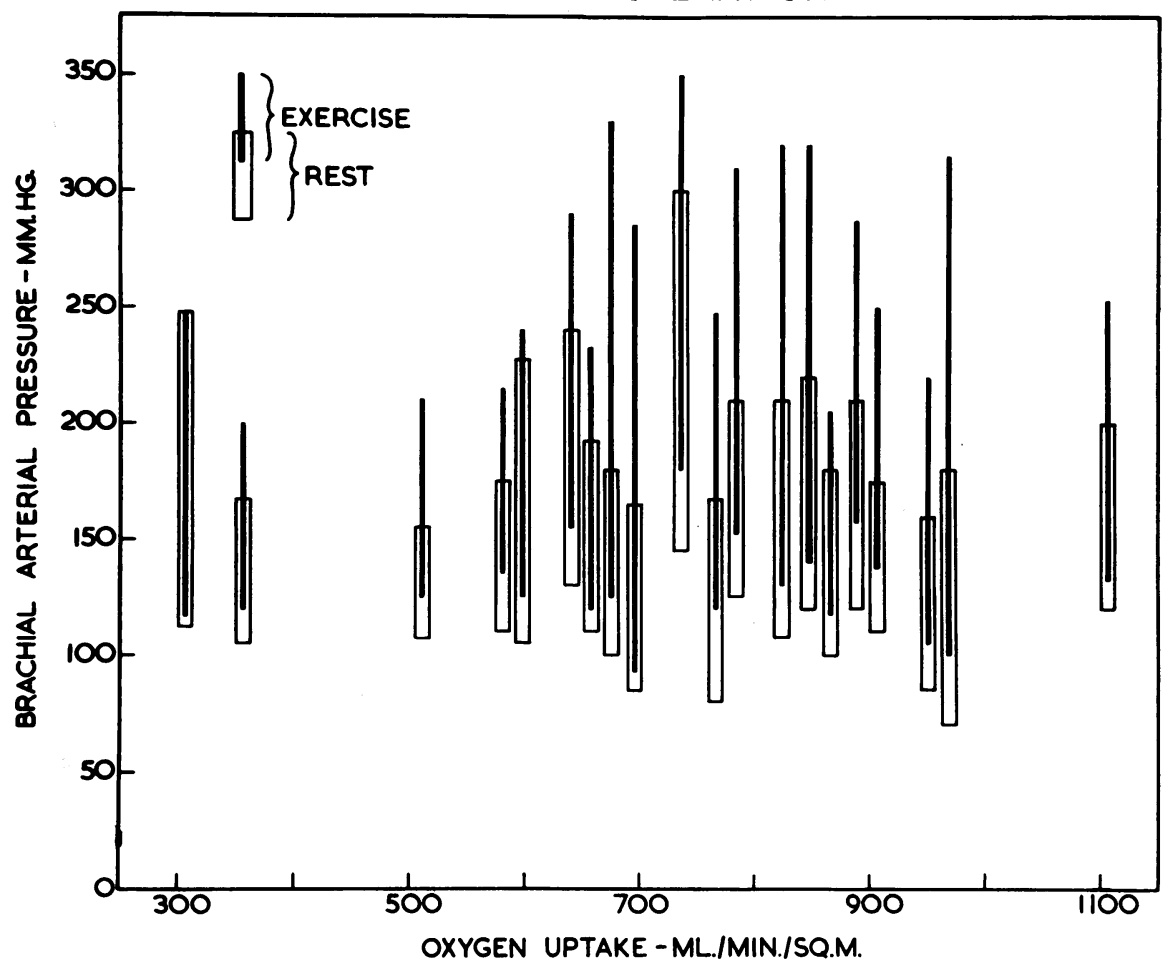

FIG. 8.-The brachial arterial pressures at rest and during exercise in 20 untreated patients with coarctation of the aorta. The resting and exercising pressures of each patient are placed at the corresponding exercising oxygen uptake.

The mean systemic arterial resistance during exercise of the 20 untreated patients was $1225 \pm 95$ dynes sec. cm. ${ }^{-5}$ (S.D. $=423$ ). In the 12 in whom the coarctation was later resected the mean exercising systemic resistance was $1406 \pm 146$ dynes sec. $\mathrm{cm} .^{-5}($ S.D. $=506)$, before and $1057 \pm 63$ dynes sec. $\mathrm{cm}^{-5}($ S.D. $=219)$ after operation $(0.05>\mathrm{P}>0.02)$. However, the mean exercising systemic arterial resistance of the 12 after operation is still significantly greater $(\mathrm{P}<0.001)$ than the value of $650 \pm 45$ dynes sec. $\mathrm{cm} .^{-5}$ (S.D. $\left.=151\right)$ for normal subjects exercising at similar levels of oxygen uptake. Of the 12 patients studied before and after operation at similar levels of oxygen uptake, one (CN1) showed a post-operative reduction in exercising systemic arterial resistance of 56 per cent of the pre-operative value and a further four (CN3, CN7, CN9, and CN10) showed post-operative reductions greater than 25 per cent. In the remainder the changes in the exercising systemic arterial resistance after operation were less.

The work of the left ventricle against pressure during exercise in the 12 patients before and after operation is related to oxygen uptake, together with the values obtained for normal subjects at similar levels of oxygen uptake in Fig. 10. Before operation the left ventricular work during exercise was increased in all. After resection of the coarctation the left ventricular work at similar levels of oxygen uptake was considerably reduced in all but two (CN1 and CN2). In four patients the post-operative left ventricular work returned to normal limits and in a further four was only moderately raised. The mean left ventricular work during exercise of the 20 untreated patients was $16.66 \pm 0.94$ (S.D. $=4 \cdot 21$ ) compared to the value of $10.29 \pm 1 \cdot 14 \mathrm{Kg} . \mathrm{m} . / \mathrm{min}$. sq.m. (S.D. $=3.78$ ) for normal subjects exercising at similar levels of oxygen uptake $(\mathrm{P}<0.001)$. 


\section{BLOOD PRESSURE AT REST AND DURING EXERCISE BEFORE AND AFTER OPERATION}

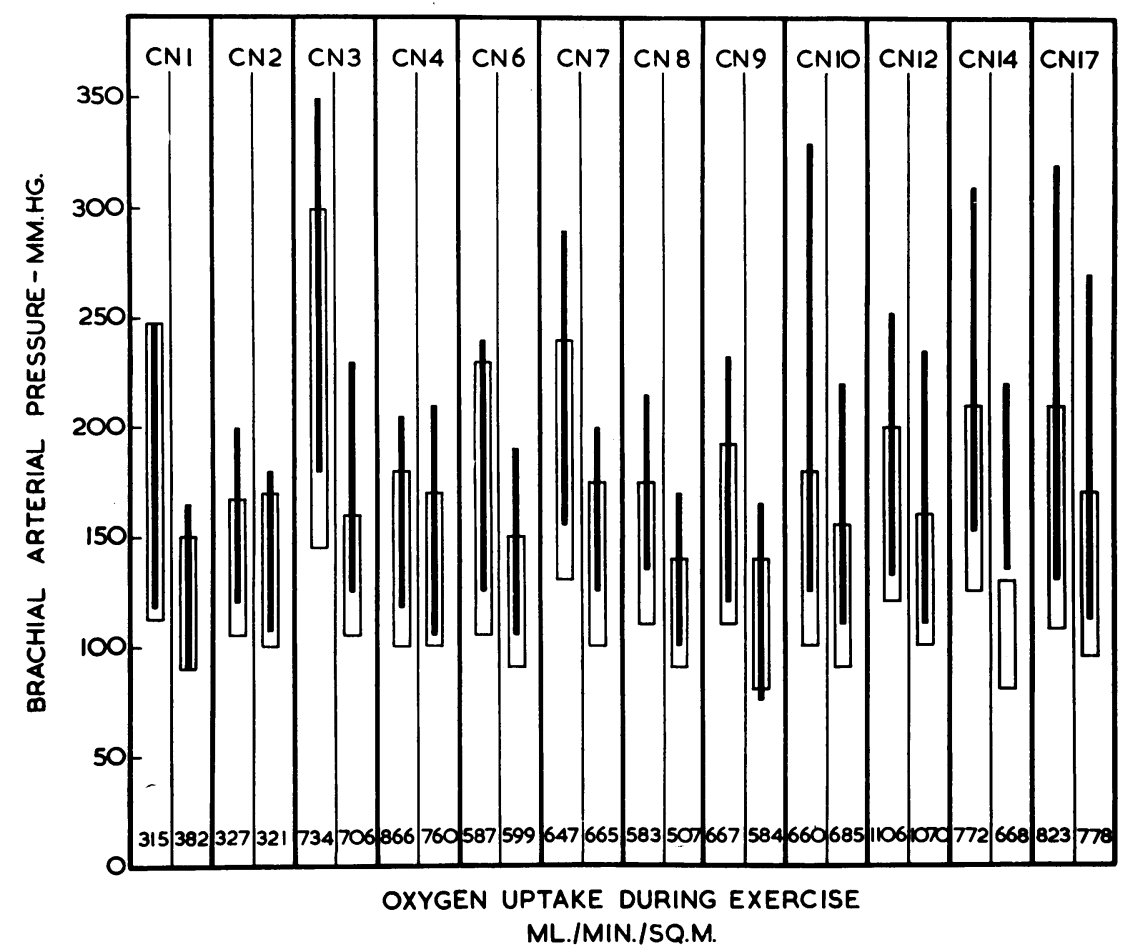

FIG. 9.-The brachial arterial pressures at rest and during exercise before and after operation compared in 12 patients. The figures at the base of each column are the exercising oxygen uptakes.

The corresponding mean work of the left ventricle during exercise in the 12 in whom the coarctation was later resected was $15.93 \pm 1.46$ (S.D.=5.06) before, and $12.43 \pm 1.03 \mathrm{Kg} . \mathrm{m} . / \mathrm{min} . / \mathrm{sq} . \mathrm{m}$. (S.D. $=3 \cdot 57)$ after operation $(0.05>\mathrm{P}>0.02)$.

\section{Discussion}

The finding of a high resting cardiac output in patients with uncomplicated coarctation of the aorta is of great interest. Calculations of the systemic vascular resistance (S.V.R.) of the upper part of the body indicate that although the raised cardiac output is not the only cause of the arterial hypertension, it certainly contributes to it in a number of instances. Six of the 20 patients studied had abnormally high resting cardiac outputs, four of these having a normal S.V.R. and two only a slightly raised S.V.R. In contrast to this, the three patients with the most severe hypertension had a normal resting cardiac output and an extremely high S.V.R. In the remaining 11 the cardiac output was raised (as compared with mean normal figures) as well as the S.V.R., both factors contributing to the rise of blood pressure in the upper part of the body (see Fig. 11.)

As the major arterial system is abnormal the systemic vascular resistance, as calculated from the brachial arterial pressure and cardiac output, cannot be employed to assess the degree of general arteriolar tone. The upper body vasculature is, as it were, in parallel with that of the lower part which has the added resistance of the coarctation and collaterals (termed "collateral resistance" hereafter) intervening between it and the upper aorta. If there were no circulatory adaptations in 
this disease the lower part of the body would receive an unduly small fraction of the cardiac output. Theoretically the lower body flow could be maintained at normal levels by three mechanisms.

First, the cardiac output could be raised so that the small fraction of the cardiac output passing to the lower body would equal the normal lower body blood flow. This would, however, result in an abnormally high blood flow to the upper body which has not been found in previous studies (Lewis, 1933; Pickering, 1935; Wakim et al., 1948; Patterson et al., 1957). Secondly, the lower body vascular resistance could be decreased in proportion to the degree of intervening collateral resistance. Patterson et al. (1957) have shown, however, that the resting vascular resistance is normal in the leg and raised in the arm, with a normal blood flow in each limb. The third mechanism, which appears to be that mainly invoked in this disease, would be an increase of upper body vascular resistance proportional to the collateral resistance. This is in accordance with the findings of Patterson et al. (1957). The demonstration of a raised resting cardiac output in some cases indicates that the first mechanism, suggested on theoretical grounds, may also be present to some degree in a number of these patients, particularly those with only a moderate increase of upper body arterial pressure.

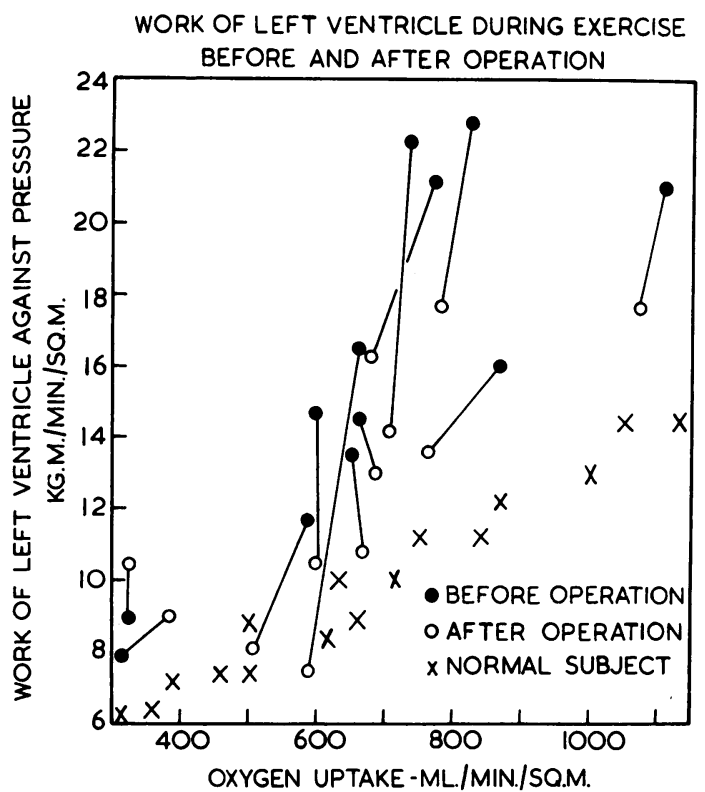

Fig. 10.-The work of the left ventricle against pressure during exercise related to oxygen uptake in 12 patients before and after operation. The values observed in normal subjects exercising at similar levels of oxygen uptake are also given (Donald et al., 1955; Donald et al., 1957).

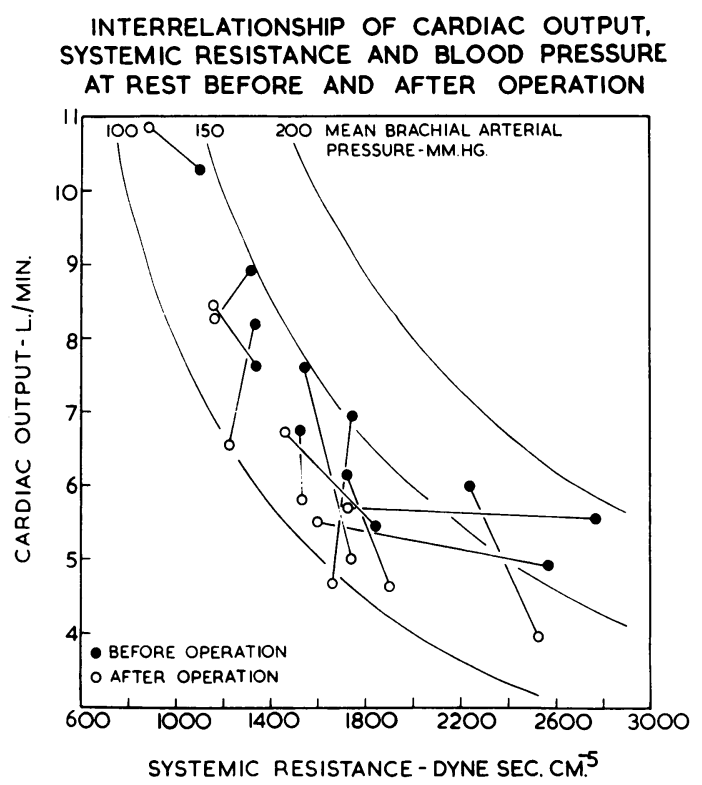

FIG. 11.-The inter-relationship of cardiac output, systemic resistance, and blood pressure at rest in 12 patients before and after operation.

The reduction in the resting brachial arterial blood pressure after operation is essentially similar to that reported by Counihan (1956). In the present study there was a large fall of the resting blood pressure after operation in all but two patients, the systolic and diastolic blood pressures of about half the group falling within the limits described by Hamilton et al. (1954) in their study of blood pressures in a hospital clinic population. In most instances, however, the figures might still be considered abnormally high, particularly in patients of this age group.

Fig. 11 shows the relative contribution of cardiac output and upper body S.V.R. to the resting brachial arterial blood pressure before and after operation. Previously it has been assumed that the fall in blood pressure after operation was due solely to a reduction of the systemic vascular 
resistance but this is not, in fact, entirely true. Ten of the 12 patients who were studied before and after operation had large or moderate reductions in the resting mean brachial arterial pressure after operation. In three, two of whom had very severe upper body hypertension, the fall in the resting brachial arterial pressure was due entirely to a reduction in the upper body S.V.R. with little change in cardiac output: in seven, the post-operative fall was due nearly exclusively to a reduction in cardiac output, the upper body S.V.R. remaining unchanged or even slightly increased. In the two patients in whom the resting brachial arterial pressure was unchanged, the post-operative reduction in the S.V.R. was countered in each case by an increase in cardiac output.

Although the establishment of a common aorta should render the compensatory vaso-constriction in the upper body no longer necessary, it is of interest that some degree of arterial hypertension often remains. If it is due to partial irreversibility of upper body vaso-constriction, functional or morphological, then the lower body vasculature must necessarily increase its resistance after operation if a normal distribution of blood to the upper and lower body is to be maintained. This interesting aspect is being pursued, as the mechanisms whereby normal or even increased regional blood flow is maintained both before and after operation should contain important clues concerning the control of the regional circulation.

The response of the brachial arterial pressure to exercise in this disease is worthy of comment, particularly as this measurement can be easily made by the clinician without any special facilities. The rise of diastolic blood pressure is not great, although it is greater than in normal subjects, particularly at higher levels of exertion. The most striking features are the enormous systolic and pulse pressures during exercise. The large pulse pressures of these patients both at rest and on exercise would appear to be due to the increased aortic diastolic pressure and decreased aortic wall compliance at such increased levels of pressure. The same phenomenon is found in essential hypertension and may be further increased by aortic wall degeneration in either disease. In coarctation of the aorta the reduced size of the immediate aortic reservoir must also contribute to the high pulse pressure.

In these studies systolic pressures of up to $350 \mathrm{~mm}$. $\mathrm{Hg}$ were recorded from the brachial artery during moderate exercise. Six out of the 20 patients investigated had exercising systolic pressures above $300 \mathrm{~mm}$. and 14 had pressures over $240 \mathrm{~mm}$. The mean brachial arterial blood pressure during exercise was also greatly raised during this relatively moderate exercise. Patients CN13 and CN16 had resting mean brachial arterial blood pressures of only 109 and $107 \mathrm{~mm}$. respectively at rest and many would consider that operating on such cases was not justifiable. However, on moderate exercise (772 and $967 \mathrm{ml} . / \mathrm{min}$./sq.m. oxygen uptake) the brachial arterial mean pressures were 163 and $172 \mathrm{~mm}$. with systolic pressures of 248 and $315 \mathrm{~mm}$. $\mathrm{Hg}$ respectively. In view of the fact that all these patients were living normal lives and exercising every day, their aortic walls would be repeatedly exposed to these very high and damaging peak systolic pressures. It would appear likely therefore that many of these patients with upper body arterial pressures that are only moderately raised at rest, who are, for the time being, in no danger of left ventricular failure, are suffering considerable damage to their aortæ and large arteries during exercise. This may well account for the striking degenerative changes found at operation in the aortic walls of patients in whom operation is delayed until left ventricular insufficiency becomes a clinical feature. It may also explain the frequent development of aortic valve regurgitation due to aortic dilatation.

Table $\mathrm{V}$ gives the mean systolic, diastolic, and mean brachial arterial pressures at rest and during exercise in the untreated patients with coarctation and in a group of 13 patients with severe essential hypertension studied previously (Taylor et al., 1957). None of these patients with essential hypertension were in clinical heart failure, but all had hypertension of such a degree that they were considered to require urgent anti-hypertensive therapy. It will be seen that the mean value of exercising systolic pressure was higher in the fit young patients with coarctation than in these patients with severe essential hypertension. Only one of the 13 with essential hypertension had a peak exercising systolic pressure over $300 \mathrm{~mm}$. compared with 6 of 20 young patients with coarctation in whom the systolic pressure was greater than $300 \mathrm{~mm}$. Hg. The patients with essential hypertension 
TABLE V

Mean Brachial Arterial Pressures at Rest and during Exercise in Coarctation of the Aorta AND ESSENTIAL HYPERTENSION

\begin{tabular}{|c|c|c|c|c|c|c|c|}
\hline & & \multicolumn{3}{|c|}{$\begin{array}{l}\text { Coarctation of aorta } \\
(20 \text { patients })\end{array}$} & \multicolumn{3}{|c|}{$\begin{array}{l}\text { Essential hypertension } \\
\quad \text { (13 patients) }\end{array}$} \\
\hline & & Systolic & Diastolic & Mean & Systolic & Diastolic & Mean \\
\hline Rest . & .. & $\begin{array}{c}198 \pm 8 \\
(36)\end{array}$ & $\begin{array}{r}107 \pm 4 \\
(18)\end{array}$ & $\begin{array}{r}138 \pm 5 \\
(22)\end{array}$ & $\begin{array}{r}205 \pm 8 \\
(28)\end{array}$ & $\begin{array}{r}125 \pm 7 \\
(24)\end{array}$ & $\begin{array}{r}151 \pm 7 \\
(24)\end{array}$ \\
\hline Exercise & .. & $\begin{array}{c}266 \pm 10 \\
(47)\end{array}$ & $\begin{array}{r}129 \pm 5 \\
(21)\end{array}$ & $\begin{array}{c}175 \pm 6 \\
(25)\end{array}$ & $\begin{array}{c}246 \pm 13 \\
(47)\end{array}$ & $\begin{array}{r}146 \pm 8 \\
(27)\end{array}$ & $\begin{array}{r}180 \pm 9 \\
(32)\end{array}$ \\
\hline
\end{tabular}

had, however, considerably higher diastolic and mean resting arterial pressures. The frequent development of cardiac or other serious complications usually does not allow these patients with severe essential hypertension, if untreated, to continue normal active lives for long periods comparable to those that can be expected in the case of many young patients with coarctation of the aorta. In addition, due to their age and frequent cardiac disability, it is unusual for such patients to take such strenuous exercise as fit young patients with coarctation. These facts allied to a normal aortic capacity in patients with essential hypertension may explain the less frequent development of aortic regurgitation in such patients.

The effect of aortic reconstruction on the exercising blood pressure was studied in 12 patients. Their pre-operative exercising blood pressures were very similar to those of the whole group of untreated patients. Operation caused a large reduction of exercising systolic, diastolic, and mean arterial blood pressure with a parallel fall of left ventricular work, allowance being made for the raised left ventricular diastolic filling pressure before operation in a number of cases. Although the mean reduction of peak systolic pressure is large in the whole group $(60 \mathrm{~mm} . \mathrm{Hg})$ it is even more striking in those with very high levels before operation. Four patients with peak systolic pressures above $300 \mathrm{~mm}$. had a mean reduction from 328 to $235(93) \mathrm{mm}$. and eight patients with exercising systolic pressures above $240 \mathrm{~mm}$. had a mean fall from 292 to $216(76) \mathrm{mm}$. $\mathrm{Hg}$. Thus, not only is left ventricular work considerably less, but the stress on the aorta is also greatly reduced. The latter consideration has not been given the attention it deserves.

The most striking finding in this investigation was the objective evidence of left ventricular insufficiency during exercise in 13 out of 20 patients, all of whom denied any significant shortness of breath on exertion. The evidence for this in all 13 was a raised wedge pressure during exercise, which is considered to be due to a raised left ventricular diastolic filling pressure under these conditions. This appears to precede reduction of the left ventricular output, as only 4 of the 13 had impaired cardiac output response to exercise and this impairment was always very small.

Another important finding was the close correlation of left ventricular behaviour and the level of the upper body mean brachial arterial pressure during exercise (Fig. 12). Of 14 patients with exercising mean brachial pressures above $160 \mathrm{~mm} ., 13 \mathrm{had}$ raised exercising wedge pressures and four of these had in addition slightly impaired exercising cardiac output responses. Of 6 patients with mean exercising blood pressures below $160 \mathrm{~mm}$., all but one had normal exercising wedge pressures and all had normal exercising cardiac outputs. In seven (CN13, CN14, CN16, CN17, $\mathrm{CN} 18, \mathrm{CN} 19$, and $\mathrm{CN} 20$ ) the exercising wedge pressure became abnormal when the mean brachial artery pressure rose above $160 \mathrm{~mm}$ although it was normal at lighter levels of exercise, when the pressure was below $160 \mathrm{~mm}$. (see Table II). Of the patients studied before and after operation, 9 of the 12 had objective evidence of left ventricular insufficiency during exercise before operation, in the form of a raised wedge pressure. After operation 8 of these 9 had normal wedge pressures and cardiac outputs on exercise: in the ninth (CN3) the raised pre-operative exercising wedge pressure persisted after operation in spite of a large reduction in brachial arterial pressure; however 
the exercising mean arterial pressure was still appreciably raised (161 mm.) even after operation. The mean fall in exercising mean arterial pressure in these eight patients who showed a return to normal left ventricular behaviour was $34 \mathrm{~mm}$. (range 7-50). Nine of the 12 patients studied had exercising brachial blood pressures greater than $160 \mathrm{~mm}$. before operation and all but three fell below this level after operation. The mean exercising pressures after operation of these three patients were 161,165 , and $165 \mathrm{~mm}$. Hg. It is of interest that a number of parents have reported a striking increase of exercise tolerance in their children after reconstruction of the aorta, although no shortness of breath or disability had been noticed before operation (d'Abreu and Parsons, 1956), It is probable that such patients have undergone hæmodynamic improvement similar to those described in this study.

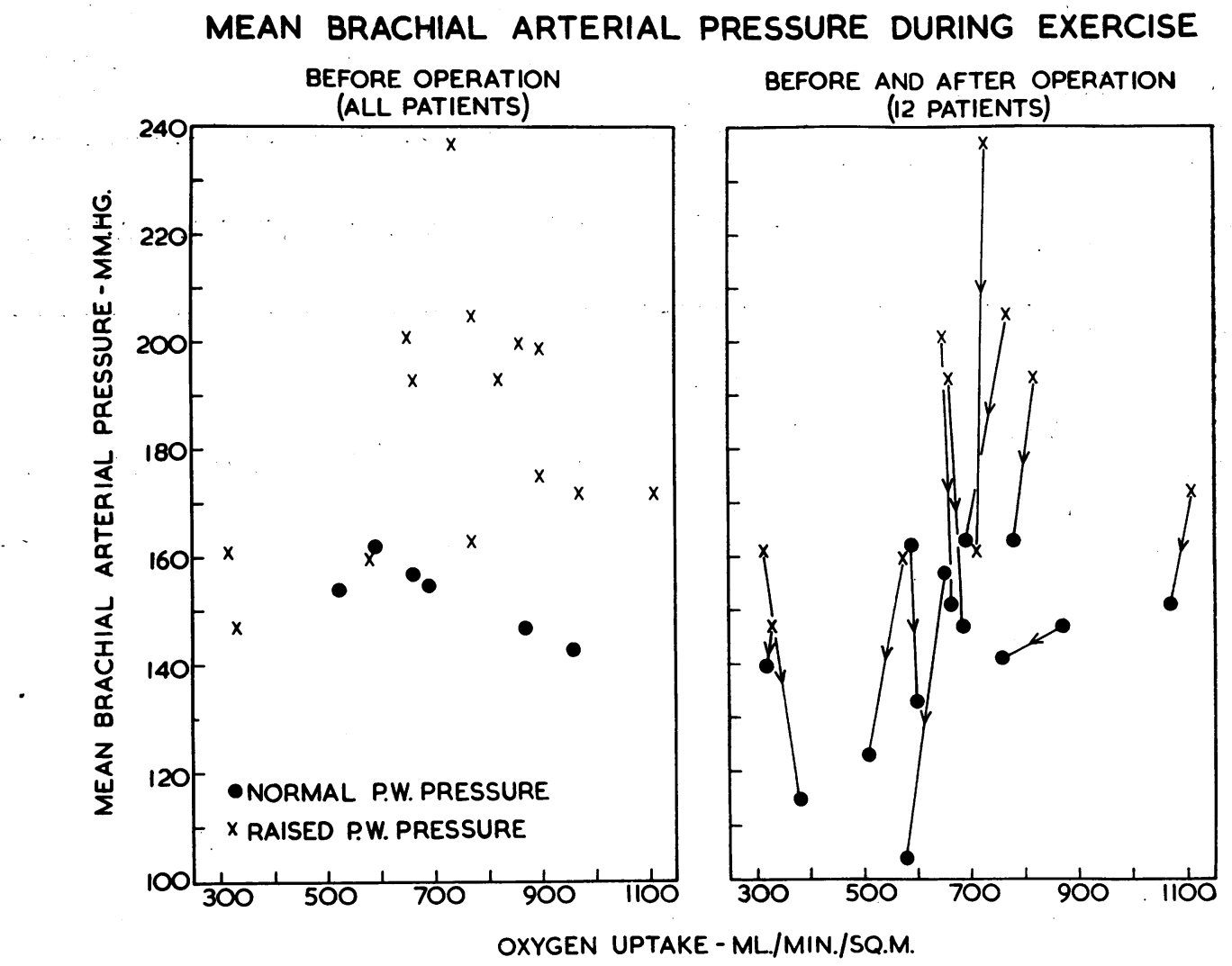

FIG. 12. - The relationship of the mean brachial arterial pressure to the pulmonary wedge pressure during exercise in 20 patients before, and in 12 of these patients after operation also.

These findings suggest that those clinicians who are in doubt as to the wisdom of operation in young persons with only slightly raised resting brachial arterial pressures would be wise to study their patients during exercise. The exercising brachial arterial pressures can easily be determined without special facilities. The data presented would indicate that if a mean exercising pressure of more than about $160 \mathrm{~mm}$. $\mathrm{Hg}$ is found, then left ventricular insufficiency, which can be remedied by aortic reconstruction, is almost certainly present. Further the finding of a very high systolic pressure on exercise may further weigh the clinical opinion in favour of operation before aortic damage renders the operation unduly hazardous.

L 


\section{SUMMARY}

Measurements of the cardiac output and intravascular pressures have been made at rest and during exercise, before and after operation, in patients with uncomplicated coarctation of the aorta.

Before operation the resting cardiac output was significantly increased as compared with normal subjects: the cardiac output response to exercise was normal. Although the pulmonary wedge pressure at rest was normal in all patients before operation, it became abnormally raised in 13 of the 20 during exercise. The large brachial arterial pulse pressure observed in all of them at rest before operation became greatly increased during exercise and in many exceeded $300 \mathrm{~mm}$. $\mathrm{Hg}$.

After operation the resting cardiac output was normal in all patients and the cardiac output response to exercise was likewise within normal limits. The resting wedge pressures were normal, but in contrast to the pre-operative observations, the exercising wedge pressures also remained within normal limits. The resting brachial arterial pressure was reduced in all but 2 of 12 patients after operation. The abnormal increase in pulse pressure during exercise still persisted.

There was a large reduction in left ventricular work both at rest and during exercise after operation. Certain hæmodynamic inter-relationships are outlined, and the significance of these findings is discussed in relation to the indications for aortic reconstruction, particularly in fit young persons without much hypertension at rest.

The authors gratefully acknowledge the co-operation readily given by Dr. O. Brenner, Prof. A. L. d'Abreu, and Mr. L. Collis whose patients were studied and the technical help of Mr. A. Pincock and Mr. R. Mills.

This work was carried out during the tenure of a Medical Research Council Fellowship in Clinical Research by one of the authors (S.H.T.). We are also indebted to grants from the Endowment Research Fund of the United Birmingham Hospitals.

\section{REFERENCES}

Beard, E. F., Wood, E. H., and Clagett, O. T. (1951). J. Lab. clin. Med., 38, 858.

Bing, R. J., Handelsmann, J. C., Campbell, J. A., Griswold, H. E., and Blalock, A. (1948). Ann. Surg., $128,803$.

Blumgart, H. L., Lawrence, J. S., and Ernstene, A. C. (1931). Arch. intern. Med., 47, 806.

Brown, G. E., Pollack, A. A., Clagett, O. T., and Wood, E. H. (1948). Proc. Staff. Meet. Mayo Clin., $23,129$.

Campbell, M. (1958). Circulation: Proceedings of the Harvey Tercentenary Congress, 1957. Edited by J. McMichael. Blackwell Scientific Publications, Oxford.

- , and Baylis, J. H. (1956). Brit. Heart J., 18, 475.

Counihan, T. B. (1956). Clin. Sci., 15, 149.

Cournand, A., Riley, R. L., Breed, E. S., Baldwin, E. de F., and Richards, D. W. (1945). J. clin. Invest., $24,106$.

Crafoord, C., and Nylin, G. (1945). J. thorac. Surg., 14, 347.

Culbertson, J. W., Eckstein, J. W., Kirkendall, W. M., and Bedell, G. N. (1957). J. clin. Invest., $36,1537$.

d'Abreu, A. L., and Parsons, C. (1956). Brit. med. J., 2, 390.

Dexter, L., Whittenberger, J. L., Haynes, F. W., Goodale, W. T., Gorlin, R., and Sawyer, C. G. (1951). J. appl. Physiol., 3, 439.

Donald, K. W., Bishop, J. M., Cumming, G., and Wade, O. L. (1953). Clin. Sci., $12,199$.

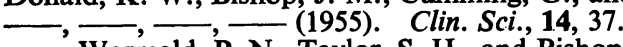

W, Wormald, P. N., Taylor, S. H., and Bishop, J. M. (1957). Clin. Sci., 16, 567.

Ebert, R. B., Borden, C. W., Wells, H. S., and Wilson, R. H. (1949). J. clin. Invest., $28,1134$.

Fisher, R. A. (1945). Statistical Methods for Research Workers, 10th ed. Oliver and Boyd, Edinburgh.

Genest, J., Newman, E. V., Kattus, A. A., Sinclair-Smith, B., and Genecin, A. (1948). Johns Hopk. Hosp. Bull., 83, 429.

Goodwin, J. F. (1958). Lancet, 1, 16.

Granström, K. O. (1951). Brit. J. Ophthalmology, 35, 143.

Graybiel, A., Allen, A. W., and White, P. D. (1935). J. clin. Invest., 14, 52.

Grollman, A., and Ferrigan, J. P., Jr. (1934). Arch. intern. Med., 53, 35.

Gross, R. E. (1953). Circulation, 7, 757.

Hallenbeck, G. A., Wood, E. H., Burchell, H. B., and Clagett, O. T. (1951). Surg. Gynec. Obstet., $92,75$.

Hamilton, M., Pickering, G. W., Roberts, J. A. F., and Sowry, G. S. C. (1954). Clin. Sci., 13, 11.

Hamilton, W. F., Riley, R. L., Attyah, A. M., Cournand, A., Fowell, A., Himmelstein, A., Noble, R. P., Remington, J. W., Richards, D. W., Wheeler, N. C., and Witham, A. C. (1948). Amer. J. Physiol., 153, 309.

Harris, J. S., Sealy, W. C., and De Maria, W. (1950). Amer. J. Med., 9, 734.

Lequime, J. (1940). Acta. med. Scand., Supplement 107.

Lewis, T. (1933). Heart, 16, 205.

Page, I. H. (1940). Amer. Heart J., 19, 218.

Patterson, G. C., Shepherd, J. T., and Whelan, R. F. (1957). Clin. Sci., 16, 627.

Pickering, G. W. (1935). Clin. Sci., 2, 209.

(1955). High Blood Pressure. Churchill, London. 
Prinzmetal, M., and Wilson, C. (1936). J. clin. Invest., 15, 63.

Stead, E. A., Warren, J. V., Merrill, A. J., and Brannon, E. S. (1945). J. clin. Invest., $24,326$.

Steele, J. M. (1941). J. clin. Invest., $20,473$.

Stewart, H. J., and Bailey, R. L., Jr. (1941). J. clin. Invest., 20, 145.

Strayhorn, W. D. (1936). Medical Papers dedicated to Henry Christian. Birthday Vol. 134. Waverly Press Inc., Baltimore.

Taylor, S. H., Donald, K. W., and Bishop, J. M. (1957). Clin. Sci., 16, 351.

Thomasson, B. (1957). Scand. J. clin. Lab. Invest., 9, 365.

Wade, O. L., Bishop, J. M., Cumming, G., and Donald, K. W. (1953). Brit. med. J., $2,902$.

Wakim, K. G., Slaughter, O., and Clagett, O. T. (1948). Proc. Mayo Clin., 23, 347.

Werkö, L., Lagerlöf, H., Bucht, H., Wehle, B., and Holmgren, A. (1949). Scand. J. clin. Lab. Invest., 1, 109.

-, Ek, J., Bucht, H., and Karnell, J. (1956). Scand. J. clin. Lab. Invest., 8, 193. 